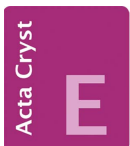

CRYSTALLOGRAPHIC COMMUNICATIONS

ISSN 2056-9890

Received 24 November 2020

Accepted 10 February 2021

Edited by A. S. Batsanov, University of Durham, England

Keywords: crystal structure; epoxyisoindole group; tetrahydrofuran ring; pyrrolidine ring; envelope conformation; boat conformation; Hirshfeld surface analysis; IMDAF reaction.

CCDC reference: 2062492

Supporting information: this article has supporting information at journals.iucr.org/e

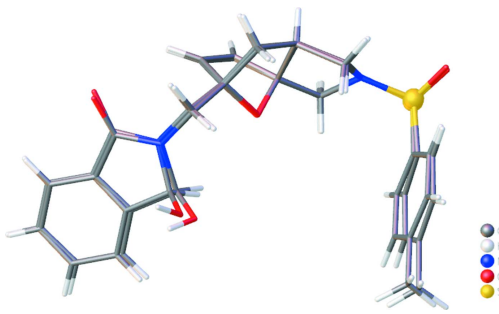

OPEN $\odot$ ACCESS

\section{Crystal structure and Hirshfeld surface analysis of (RS)-3-hydroxy-2-\{[(3aRS,6RS,7aRS)-2-(4-methyl- phenylsulfonyl)-2,3,3a,6,7,7a-hexahydro-3a,6-ep- oxy-1H-isoindol-6-yl]methyl\}isoindolin-1-one}

Dmitriy F. Mertsalov, a Maryana A. Nadirova, ${ }^{a}$ Elena A. Sorokina, ${ }^{a}$ Marina A. Vinokurova, ${ }^{a}$ Sevim Türktekin Celikesir, ${ }^{b}$ Mehmet Akkurt, ${ }^{b}$ Irina A. Kolesnik ${ }^{c}$ and Ajaya Bhattarai ${ }^{d_{*}}$

a'Department of Organic Chemistry, Peoples' Friendship University of Russia, (RUDN University), 6 Miklukho-Maklaya St., 117198, Moscow, Russian Federation, b Department of Physics, Faculty of Sciences, Erciyes University, 38039 Kayseri, Turkey, ' Laboratory of Organoelement Compounds, Institute of Physical Organic Chemistry, National Academy of Sciences of Belarus, 13 Surganov St., 220072, Minsk, Belarus, and ${ }^{\mathbf{d}}$ Department of Chemistry, M.M.A.M.C (Tribhuvan University), Biratnagar, Nepal. *Correspondence e-mail: bkajaya@yahoo.com

The title compound, $\mathrm{C}_{24} \mathrm{H}_{24} \mathrm{~N}_{2} \mathrm{O}_{5} \mathrm{~S}$, crystallizes with two independent molecules $(A$ and $B)$ in the asymmetric unit. In the central ring systems of both molecules, the tetrahydrofuran rings adopt envelope conformations, the pyrrolidine rings adopt a twisted-envelope conformation and the six-membered ring is in a boat conformation. In molecules $A$ and $B$, the nine-membered groups attached to the central ring system are essentially planar (r.m.s. deviations of 0.002 and $0.003 \AA$, respectively). They form dihedral angles of $64.97(9)$ and $56.06(10)^{\circ}$, respectively, with the phenyl rings. In the crystal, strong intermolecular $\mathrm{O}-$ $\mathrm{H}$. . O hydrogen bonds and weak intermolecular $\mathrm{C}-\mathrm{H} \cdots \mathrm{O}$ contacts link the molecules, forming a three-dimensional network. In addition weak $\pi-\pi$ stacking interactions [centroid-to centroid distance $=3.7124(13) \AA$ ] between the pyrrolidine rings of the nine-membered groups of $A$ molecules are observed. Hirshfeld surface analysis and two-dimensional fingerprint plots were used to quantify the intermolecular interactions present in the crystal, indicating that the environments of the two molecules are very similar. The most important contributions for the crystal packing are from $\mathrm{H} \cdots \mathrm{H}(55.8 \%$ for molecule $A$ and $53.5 \%$ for molecule $B), \mathrm{O} \cdots \mathrm{H} / \mathrm{H} \cdots \mathrm{O}(24.5 \%$ for molecule $A$ and $26.3 \%$ for molecule $B$ ) and $\mathrm{C} \cdots \mathrm{H} / \mathrm{H} \cdots \mathrm{C}(12.6 \%$ for molecule $A$ and $15.7 \%$ for molecule $B)$ interactions.

\section{Chemical context}

Currently, considerable attention is being paid to the development of atom- and step-economic tools in order to obtain new, practically useful materials. Tandem and domino reactions play an important role in this arsenal, since the isolation of intermediates is not required in these processes, as all reaction steps occur spontaneously (Tietze \& Beifuss, 1993).

As an example of using such synthetic tools, we proposed the synthesis of compound $\mathbf{3}$, which contains three privileged scaffolds, based on the tandem Hinsberg/IMDAF (intramolecular Diels-Alder furan; Zubkov et al., 2005, 2014) reaction strategy (Demircan et al., 2016; Nadirova et al., 2020). Substituted sulfonamides are important because of their broad spectrum of biological activities (Anderson et al., 2012) while 3-hydroxyisoindol-1-ones are well-known nitrogen-containing heterocyclic compounds with a wide range of physiological activity: agonists of muscarinic M2 receptor modulators, 
antimicrobial activity etc. (Stiefl et al., 2003; Breytenbach et al., 2000).

The reaction proceeds smoothly in boiling water. Separation and subsequent crystallization of the resulting solids from ethyl acetate provides the title adduct $\mathbf{3}$ in moderate yield. The process starts with the Hinsberg $N$-sulfonylation of amine $\mathbf{1}$, leading to the formation of the intermediate $N$-sulfonamide (2), which undergoes spontaneous intramolecular Diels-Alder reaction. It should be noted that the exo-[4+2] cycloaddition proceeds stereoselectively with the exclusive formation of diastereoisomer 3 (Fig. 1).

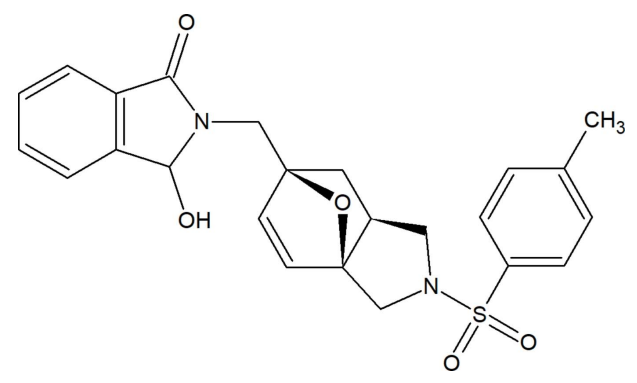

On the other hand, non-covalent interactions between molecules play an important role in the synthesis, crystal engineering, molecular recognition, and as key activating/ controlling elements in the field of catalysis (Afkhami et al., 2017; Asadov et al., 2016; Gurbanov et al., 2017, 2018; Karmakar et al., 2017; Kopylovich et al., 2011a,b; Ma et al., 2017a,b; Maharramov et al., 2018; Mahmoudi et al., 2017, 2019; Mahmudov et al., 2010, 2020; Mizar et al., 2012; Sutradhar et al., 2015). Herein, we highlight the role of weak interactions in the structural features of molecule 3 .

\section{Structural commentary}

As shown Fig. 2, the title compound $\mathbf{3}$ crystallizes with two independent molecules ( $A$ with the atom $\mathrm{S}$ and $B$ with the atom $\mathrm{S}^{\prime}$ ) in the asymmetric unit in which the epoxyisoindole and phenyl rings are linked through an $\mathrm{N}-\mathrm{S}-\mathrm{C}$ bridge. In the central ring systems of molecules $A$ and $B$, the two tetrahydrofuran rings $(A$ : $\mathrm{O} 3 / \mathrm{C} 10-\mathrm{C} 13, \mathrm{O} 3 / \mathrm{C} 10 / \mathrm{C} 13 / \mathrm{C} 15 / \mathrm{C} 16$ and B: $\left.\mathrm{O}^{\prime} / \mathrm{C} 10^{\prime}-\mathrm{C}^{\prime} 3^{\prime}, \mathrm{O}^{\prime} / \mathrm{C}^{\prime} 0^{\prime} / \mathrm{C}^{\prime} 3^{\prime} / \mathrm{C} 15^{\prime} / \mathrm{C} 16^{\prime}\right)$ adopt envelope conformations [puckering parameters (Cremer \& Pople, 1975) $Q=0.508$ (2), 0.600 (2) and 0.523 (2), 0.602 (2) $\AA$, respectively], the pyrrolidine rings $(A$ : N2/C13-C14/C16-C17 and $B$ : $\left.\mathrm{N} 2^{\prime} / \mathrm{C} 13^{\prime}-\mathrm{C} 14^{\prime} / \mathrm{C} 16^{\prime}-\mathrm{C} 17^{\prime}\right)$ adopt a twisted-envelope confor-

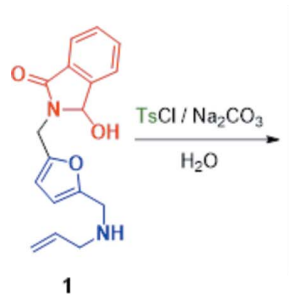

1

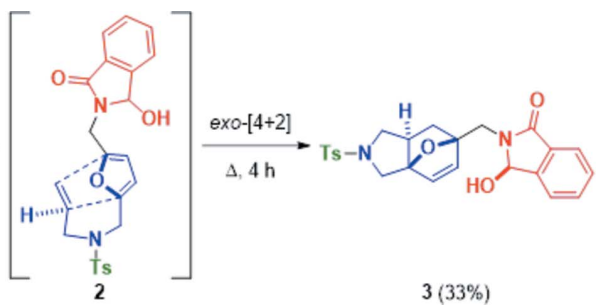

$3(33 \%)$
Figure 1

Synthesis of the title compound $\mathbf{3}$.

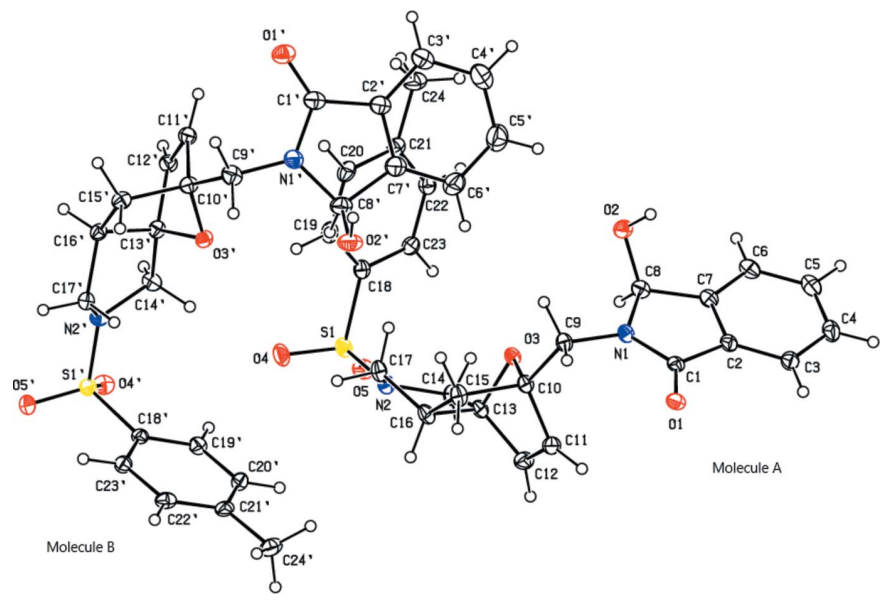

Figure 2

View of the two independent molecules, $A$ and $B$, in the asymmetric unit of the title compound 3, with displacement ellipsoids for the nonhydrogen atoms drawn at the $30 \%$ probability level.

mation $\left[Q_{\mathrm{T}}=0.392(2) \AA, \varphi(2)=132.8(4)^{\circ}\right.$ and $Q_{\mathrm{T}}=$ $0.408(2) \AA, \varphi(2)=310.0(3)^{\circ}$, respectively] and the sixmembered rings are in a boat conformation (C10-C13/C15/ C16; $Q_{\mathrm{T}}=0.965(2) \AA, \theta=89.90(12)^{\circ}, \varphi=180.80(15)^{\circ}$ in

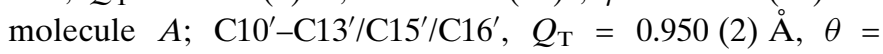
$89.90(12)^{\circ}, \varphi=0.57(15)^{\circ}$ in molecule $B$ ].

In molecules $A$ and $B$, the nine-membered groups ( $A: \mathrm{N} 1 /$ $\mathrm{C} 1-\mathrm{C} 8$ and $\left.B: \mathrm{N}^{\prime} / \mathrm{C}^{\prime}-\mathrm{C}^{\prime}\right)$ attached to the central ring system are essentially planar (r.m.s deviations of 0.002 and $0.003 \AA$, respectively). They form dihedral angles of 64.97 (9) and $56.06(10)^{\circ}$, respectively, with the phenyl rings ( $A$ : $\mathrm{C} 18-\mathrm{C} 23$ and $\left.B: \mathrm{C} 18^{\prime}-\mathrm{C} 23^{\prime}\right)$. Fig. 3 shows the overlay of molecules $A$ and $B$ in the asymmetric unit (r.m.s. deviation $0.252 \AA$ ).

\section{Supramolecular features}

In the crystal, strong intermolecular $\mathrm{O}-\mathrm{H} \cdots \mathrm{O}$ hydrogen bonds and weak intermolecular $\mathrm{C}-\mathrm{H}$... O contacts link the molecules, forming a three-dimensional network (Table 1, Fig. 4). In addition weak $\pi-\pi$ stacking interactions are

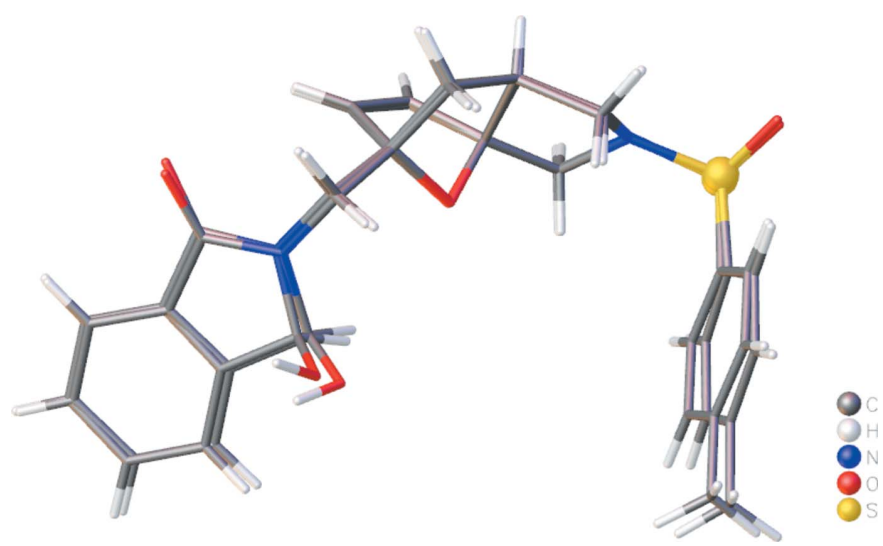

Figure 3

Overlay image of the two molecules $(A$ and $B)$ in the asymmetric unit of the title compound $\mathbf{3}$. 
Table 1

Hydrogen-bond geometry $\left(\AA,^{\circ}\right)$.

\begin{tabular}{lllll}
\hline$D-\mathrm{H} \cdots A$ & $D-\mathrm{H}$ & $\mathrm{H} \cdots A$ & $D \cdots A$ & $D-\mathrm{H} \cdots A$ \\
\hline $\mathrm{C} 12-\mathrm{H} 12 \cdots \mathrm{O}^{\mathrm{i}}$ & 0.95 & 2.47 & $3.333(3)$ & 151 \\
$\mathrm{C} 14-\mathrm{H} 14 A \cdots 5^{\prime \text { ii }}$ & 0.99 & 2.62 & $3.506(3)$ & 149 \\
$\mathrm{C} 23-\mathrm{H} 23 \cdots 5^{\prime \text { ii }}$ & 0.95 & 2.41 & $3.195(3)$ & 139 \\
$\mathrm{C} 14^{\prime}-\mathrm{H} 14 D \cdots \mathrm{O} 4$ & 0.99 & 2.58 & $3.482(3)$ & 151 \\
$\mathrm{C} 15^{\prime}-\mathrm{H} 15 C \cdots \mathrm{O} 2^{\mathrm{iii}}$ & 0.99 & 2.62 & $3.585(3)$ & 165 \\
$\mathrm{C} 16^{\prime}-\mathrm{H} 16^{\prime} \cdots \mathrm{O} 4^{\prime \text { iv }}$ & 1.00 & 2.53 & $3.422(3)$ & 149 \\
$\mathrm{C} 19^{\prime}-\mathrm{H} 19^{\prime} \cdots \mathrm{O} 4$ & 0.95 & 2.34 & $3.078(3)$ & 134 \\
$\mathrm{O} 2-\mathrm{H} 2 \cdots{ }^{\mathrm{v}}$ & 0.92 & 1.85 & $2.756(2)$ & 172 \\
$\mathrm{O}^{\prime}-\mathrm{H} 2^{\prime} \cdots \mathrm{O}^{\prime \text { vi }}$ & 0.90 & 1.95 & $2.840(3)$ & 171 \\
\hline
\end{tabular}

Symmetry codes: (i) $-x+1,-y+1,-z+1$; (ii) $x, y-1, z$; (iii) $x, y+1, z$; (iv) $-x+2,-y+2,-z+1 ;(\mathrm{v})-x+1,-y,-z+2 ;(\mathrm{vi})-x+2,-y+1,-z+2$.

observed $[C g 3 \cdots C g 3(1-x,-y, 2-z)=3.7124$ (13) A where $\mathrm{Cg} 3$ is the centroid of the pyrrolidine ring $(\mathrm{N} 1 / \mathrm{C} 1 / \mathrm{C} 2 / \mathrm{C} 7 / \mathrm{C} 8)$ of the nine-membered group in molecule $A$, with slippage of $1.675 \AA$ ] .

\section{Hirshfeld surface analysis}

The Hirshfeld surfaces for both independent molecules ( $A$ and $B)$ in the asymmetric of the title compound $\mathbf{3}$ were generated using Crystal Explorer 17 (Turner et al., 2017). The $d_{\text {norm }}$ mappings were performed in the range of -0.6446 to 1.7383 arbitrary units for the molecule $A$ and -0.5749 to 1.6904 arbitrary units for molecule $B$. Bold red circles on the $d_{\text {norm }}$ surfaces (Fig. 5a) indicate regions of $\mathrm{O}-\mathrm{H} \cdots \mathrm{O}$ interactions. The $\mathrm{C}-\mathrm{H}$... O interactions also cause red spots on the Hirshfeld surfaces. The shape-index maps (Fig. $5 b$ ) contain red and blue triangles related to $\pi-\pi$ interactions.

Fingerprint plots (Fig. 6) reveal that while $\mathrm{H} \cdots \mathrm{H}(55.8 \%$ for molecule $A$ and $53.5 \%$ for molecule $B$ ) interactions make

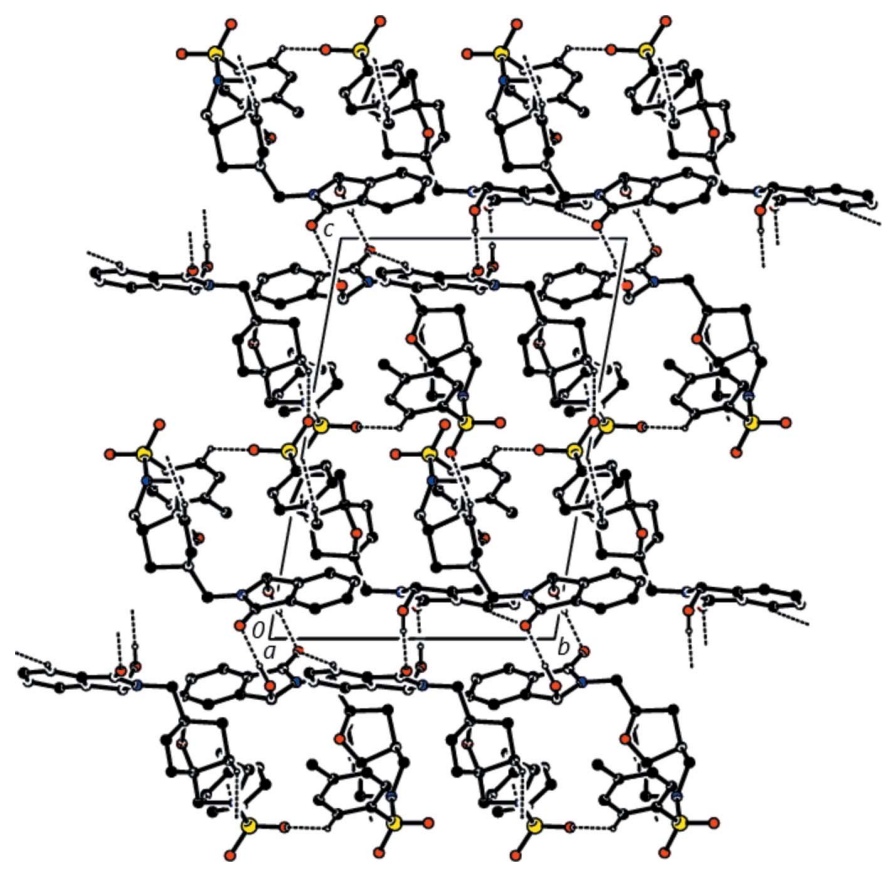

Figure 4

A view of the intermolecular $\mathrm{C}-\mathrm{H} \cdots \mathrm{O}$ and $\mathrm{O}-\mathrm{H} \cdots \mathrm{O}$ interactions in the crystal structure of the title compound $\mathbf{3}$.
Table 2

Summary of short interatomic contacts $(\AA)$ in the title compound $\mathbf{3}$.

\begin{tabular}{lll}
\hline Contact & Distance & Symmetry operation \\
\hline $\mathrm{O} 1 \cdots \mathrm{H} 3^{\prime}$ & 2.27 & $-1+x, y, z$ \\
$\mathrm{H} 2 \cdots \mathrm{O} 1$ & 1.85 & $1-x,-y, 2-z$ \\
$\mathrm{O} 4 \cdots \mathrm{H} 19^{\prime}$ & 2.34 & $x, y, z$ \\
$\mathrm{H} 8 \cdots \mathrm{H} 17 C$ & 2.41 & $x,-1+y, z$ \\
$\mathrm{H} 12 \cdots \mathrm{O} 5$ & 2.47 & $1-x, 1-y, 1-z$ \\
$\mathrm{C} 20 \cdots \mathrm{O} 5$ & 2.411 & $2-x, 1-y, 1-z$ \\
$\mathrm{H} 11 \cdots \mathrm{H} 24 B$ & 2.43 & $-1+x, y, z$ \\
$\mathrm{H} 15 B \cdots \mathrm{H} 5$ & 2.42 & $x, 1+y, z$ \\
$\mathrm{H} 22 \cdots \mathrm{H} 14 C$ & 2.23 & $2-x, 1-y, 1-z$ \\
$\mathrm{H} 2^{\prime} \cdots 1^{\prime}$ & 1.95 & $2-x, 1-y, 2-z$ \\
$\mathrm{H} 24 F \cdots 4^{\prime}$ & 2.40 & $2-x, 2-y, 1-z$ \\
$\mathrm{H} 16^{\prime} \cdots 4^{\prime}$ & 2.53 & $1+x, y, z, 1-z$ \\
$\mathrm{H} 16^{\prime} \cdots \mathrm{H} 24 F$ & 2.41 & \\
\hline
\end{tabular}

Table 3

Percentage contributions of interatomic contacts to the Hirshfeld surfaces for molecules $A$ and $B$ of the title compound $\mathbf{3}$.

\begin{tabular}{lll}
\hline & Molecule $A$ & Molecule $B$ \\
\hline Contact & Percentage contribution & Percentage contribution \\
$\mathrm{H} \cdots \mathrm{H}$ & 55.8 & 53.5 \\
$\mathrm{O} \cdots \mathrm{H} / \mathrm{H} \cdots \mathrm{O}$ & 24.5 & 26.3 \\
$\mathrm{C} \cdots \mathrm{H} / \mathrm{H} \cdots \mathrm{C}$ & 12.6 & 15.7 \\
$\mathrm{C} \cdots \mathrm{C}$ & 3.3 & 2.6 \\
$\mathrm{C} \cdots \mathrm{O} / \mathrm{O} \cdots \mathrm{C}$ & 2.6 & 0.4 \\
$\mathrm{~N} \cdots \mathrm{H} / \mathrm{H} \cdots \mathrm{N}$ & 0.8 & 1.2 \\
$\mathrm{C} \cdots \mathrm{N} / \mathrm{N} \cdots \mathrm{C}$ & 0.5 & 0.1 \\
$\mathrm{~N} \cdots \mathrm{O} / \mathrm{O} \cdots \mathrm{N}$ & - & 0.1 \\
$\mathrm{~S} \cdots \mathrm{H} / \mathrm{H} \cdots \mathrm{S}$ & - & 0.1 \\
$\mathrm{~S} \cdots \mathrm{H} / \mathrm{H} \cdots \mathrm{S}$ & 0.1 & - \\
\hline
\end{tabular}

the greatest contributions to the surface contacts (Table 2), as would be expected for a molecule with such a predominance of $\mathrm{H}$ atoms, $\mathrm{O} \cdots \mathrm{H} / \mathrm{H} \cdots \mathrm{O}(24.5 \%$ for molecule $A$ and $26.3 \%$ for molecule $B$ ) and C $\cdots \mathrm{H} / \mathrm{H} \cdots \mathrm{C}(12.6 \%$ for molecule $A$ and $15.7 \%$ for molecule $B$ ) contacts are also substantial. Table 3
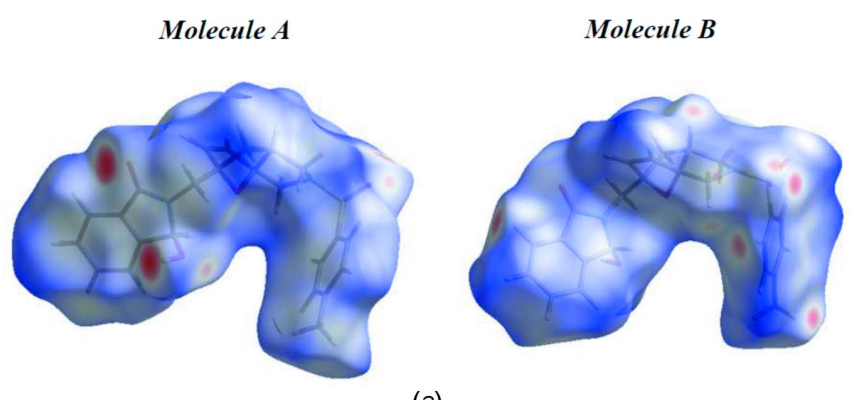

(a)
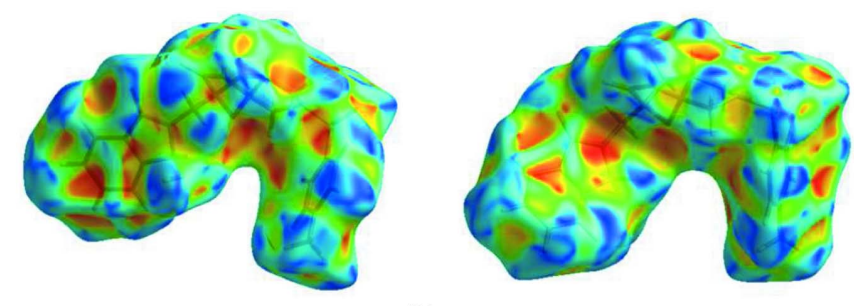

(b)

Figure 5

(a) View of the three-dimensional Hirshfeld surfaces for molecules $A$ and $B$ of the title compound 3 ; $(b)$ Hirshfeld surfaces plotted over shapeindex. 

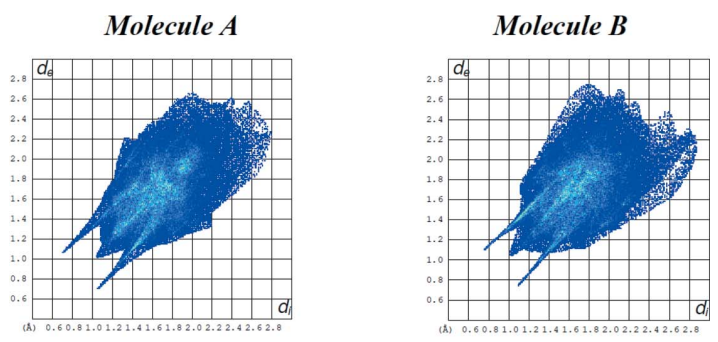

(a) All...All
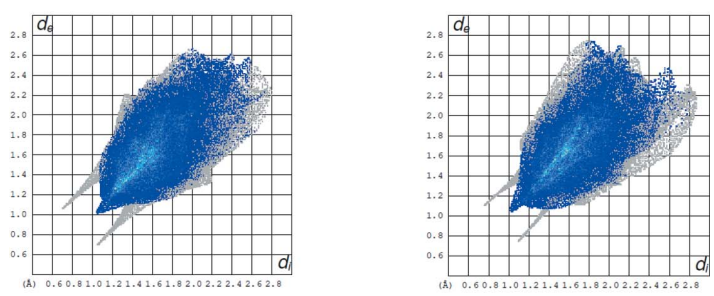

(b) $H$...H
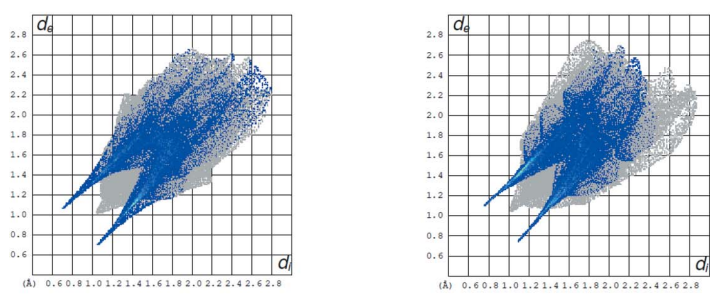

(c) $O \ldots H / H \ldots O$
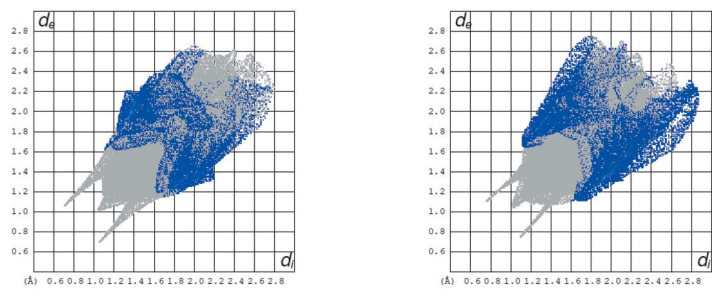

(d) $C$...H $/ H \ldots C$

Figure 6

The two-dimensional fingerprint plots for molecules $A$ and $B$ of the title compound 3 showing $(a)$ all interactions, and delineated into $(b) \mathrm{H} \cdots \mathrm{H}$, (c) $\mathrm{O} \cdots \mathrm{H} / \mathrm{H} \cdots \mathrm{O}$ and $(d) \mathrm{C} \cdots \mathrm{H} / \mathrm{H} \cdots \mathrm{C}$ interactions. The $d_{\mathrm{i}}$ and $d_{\mathrm{e}}$ values are the closest internal and external distances (in $\AA$ ) from given points on the Hirshfeld surface.

gives the contributions of the other, less significant contacts. As shown in Table 3, the environments of the two molecules $A$ and $B$ are very similar. Even the packing looks pseudomonoclinic, with a pseudo-glide plane relating the two molecules $A$ and $B$.

\section{Database survey}

There are several examples of structures closely related to the 2 -(dioxo- $\lambda$ 6-sulfanyl)octahydro-3a,6-epoxyisoindole skeleton of 3. Selected examples found in the Cambridge Structural Database (CSD, version 5.40, update of August 2019; Groom et al., 2016) include ( $3 \mathrm{a} R, 6 S, 7 \mathrm{a} R)$-7a-bromo-2-methylsulfonyl1,2,3,6,7,7a-hexahydro-3a,6-epoxyisoindole (CSD refcode ERIVIL; Temel et al., 2011), (3aR,6S,7aR)-7a-chloro-2-[(4- nitrophenyl)sulfonyl]-1,2,3,6,7,7a-hexahydro-3a,6-epoxyisoindole (AGONUH; Temel et al., 2013), (3aR,6S,7aR)-7achloro-6-methyl-2-[(4-nitrophenyl)sulfonyl]-1,2,3,6,7,7a-hexahydro-3a,6-epoxyisoindole (TIJMIK; Demircan et al., 2013), (3aR,6S,7a $R)$-7a-bromo-2-[(4-methylphenyl)sulfonyl]-1,2,3,6,7,7a-hexahydro-3a,6-epoxyisoindole (UPAQEI; Koşar et al., 2011), 5-chloro-7-methyl-3-[(4-methylphenyl)sulfonyl]-10oxa-3-azatricyclo[5.2.1.01,5]dec-8-ene (YAXCIL; Temel et al., 2012), tert-butyl 3a-chloroperhydro-2,6a-epoxyoxireno(e)isoindole-5-carboxylate (MIGTIG; Koşar et al., 2007) and 2-(2-aminoethyl)-3a,4,7,7a-tetrahydro-1 $H$-4,7-epoxyisoindole1,3(2H)-dione (BILLAL; Mitchell et al., 2013).

In the crystal of ERIVIL, weak intermolecular $\mathrm{C}-\mathrm{H} \cdots \mathrm{O}$ hydrogen bonds link the molecules into $R_{2}^{2}(8)$ and $R_{2}^{2}(14)$ rings along the $b$-axis direction. In the crystal of AGONUH, C$\mathrm{H}$... O hydrogen bonds link the molecules into zigzag chains running along the $b$-axis direction. In the crystal of TIJMIK, two types of $\mathrm{C}-\mathrm{H} \cdots \mathrm{O}$ hydrogen bonds generate $R_{2}^{2}(20)$ and $R_{4}^{4}(26)$ rings, with adjacent rings running parallel to the $a c$ plane. Further $\mathrm{C}-\mathrm{H} \cdots \mathrm{O}$ hydrogen bonds form a $C(6)$ chain, linking the molecules in the $b$-axis direction. In the crystal of UPAQEI, molecules are linked by $\mathrm{C}-\mathrm{H} \cdots \mathrm{O}$ hydrogen bonds. In the crystal of YAXCIL, $\mathrm{C}-\mathrm{H} \cdots \mathrm{O}$ hydrogen bonds link the molecules into a three-dimensional network. In the crystal of MIGTIG, the molecules are linked only by weak van der Waals interactions. The compound BILLAL contains two molecules in the asymmetric unit, which are hydrogen-bonded dimers. The bonds closest to linearity are between the carbonyl groups and the amine $\mathrm{H}$ atoms. Intermolecular hydrogen bonding involving the $\mathrm{O}$ atoms also occurs.

\section{Synthesis and crystallization}

4-Toluenesulfonyl chloride $(0.61 \mathrm{~g}, 3.2 \mathrm{mmol})$ was added to 2(\{5-[(allylamino)methyl]-2-furyl\}methyl)-3-hydroxyisoindolin1-one $(0.79 \mathrm{~g}, 2.7 \mathrm{mmol})$ in water $(10 \mathrm{~mL})$ in the presence of $\mathrm{Na}_{2} \mathrm{CO}_{3}(0.34 \mathrm{~g}, 3.2 \mathrm{mmol})$. The resulting reaction mixture was refluxed for $4 \mathrm{~h}$ and then extracted with DCM $(3 \times 10 \mathrm{~mL})$. The organic layers were dried with anhydrous $\mathrm{MgSO}_{4}$. The desiccator was filtered off, the solution concentrated and the residue was recrystallized from EtOAc. The obtained precipitate was filtered off, washed with hexane $(3 \times 5 \mathrm{~mL})$ and dried in air to give $0.4 \mathrm{~g}$ (33\%) of (RS)-3-hydroxy-2$\{[(3 \mathrm{a} R S, 6 R S, 7 \mathrm{a} R S)-2-(4-m e t h y l p h e n y l s u l f o n y l)-2,3,3 \mathrm{a}, 6,7,7 \mathrm{a}-$ hexahydro-3a,6-epoxy-1H-isoindol-6-yl]methyl lisoindolin-1one $(3)$ as colourless prisms, m.p. $=468.1-469.1 \mathrm{~K} . R_{\mathrm{f}}=0.6$ (EtOH-DMF, 1:2). IR (KBr), $v\left(\mathrm{~cm}^{-1}\right): 1167\left(v_{\mathrm{s}} \mathrm{SO}_{2}\right), 1340$ $\left(v_{\mathrm{as}} \mathrm{SO}_{2}\right), 1679(\mathrm{NCO}), 3281(\mathrm{OH}) .{ }^{1} \mathrm{H}$ NMR (DMSO- $d_{6}$, $400 \mathrm{MHz}, 301 \mathrm{~K}): \delta=7.74-7.43(m, 8 \mathrm{H}, \mathrm{HAr}), 6.41(d, 1 \mathrm{H}, \mathrm{OH}$, $J=9.3), 6.37$ and $6.22(2 d, 2 \mathrm{H}, \mathrm{H} 4, \mathrm{H} 5, J=5.7), 5.68(d, 1 \mathrm{H}$, CH-O, $J=9.3), 4.20\left(d, 1 \mathrm{H}, \mathrm{NCH}_{2} A, J=15.3\right), 3.78(d, 1 \mathrm{H}$, $\mathrm{H} 3 A, J=12.1), 3.73(t, 1 \mathrm{H}, \mathrm{H}-1 A, J=9.5), 3.52\left(d, 1 \mathrm{H}, \mathrm{NCH}_{2} B\right.$, $J=15.3), 3.42(d, 1 \mathrm{H}, \mathrm{H} 3 B, J=12.1), 2.79(t, 1 \mathrm{H}, \mathrm{H}-1 B, J=9.5)$, $2.43\left(s, 3 \mathrm{H}, \mathrm{CH}_{3}\right), 2.00-1.93(m, 1 \mathrm{H}, \mathrm{H} 7 A), 1.55-1.44(m, 2 \mathrm{H}$, H7). ${ }^{13} \mathrm{C}$ NMR (DMSO- $d_{6}, 100.4 \mathrm{MHz}, 301 \mathrm{~K}$ ): $\delta=166.2$, 145.0, 143.4, 137.6, 135.5, 133.9, 132.0, 131.1, 129.8, 129.2, 127.2, 
Table 4

Experimental details.

\begin{tabular}{|c|c|}
\hline Crystal data & \\
\hline Chemical formula & $\mathrm{C}_{24} \mathrm{H}_{24} \mathrm{~N}_{2} \mathrm{O}_{5} \mathrm{~S}$ \\
\hline$M_{\mathrm{r}}$ & 452.51 \\
\hline Crystal system, space group & Triclinic, $P \overline{1}$ \\
\hline Temperature $(\mathrm{K})$ & 120 \\
\hline$a, b, c(\AA)$ & $\begin{array}{l}11.8210(8), 11.8395(8) \\
16.7336(11)\end{array}$ \\
\hline$\alpha, \beta, \gamma\left({ }^{\circ}\right)$ & 77.949 (1), 79.555 (1), 77.511 (1) \\
\hline$V\left(\AA^{3}\right)$ & $2213.3(3)$ \\
\hline$Z$ & 4 \\
\hline Radiation type & Мо $K \alpha$ \\
\hline$\mu\left(\mathrm{mm}^{-1}\right)$ & 0.19 \\
\hline Crystal size $(\mathrm{mm})$ & $0.15 \times 0.09 \times 0.06$ \\
\hline Data collection & \\
\hline Diffractometer & Bruker APEXII CCD \\
\hline Absorption correction & $\begin{array}{l}\text { Multi-scan (SADABS; Bruker, } \\
\text { 2013) }\end{array}$ \\
\hline$T_{\min }, T_{\max }$ & $0.688,0.746$ \\
\hline $\begin{array}{l}\text { No. of measured, independent and } \\
\text { observed }[I>2 \sigma(I)] \text { reflections }\end{array}$ & $29692,13532,8799$ \\
\hline$R_{\text {int }}$ & 0.043 \\
\hline$(\sin \theta / \lambda)_{\max }\left(\AA^{-1}\right)$ & 0.717 \\
\hline Refinement & \\
\hline$R\left[F^{2}>2 \sigma\left(F^{2}\right)\right], w R\left(F^{2}\right), S$ & $0.060,0.147,1.02$ \\
\hline No. of reflections & 13532 \\
\hline No. of parameters & 581 \\
\hline H-atom treatment & $\begin{array}{l}\mathrm{H} \text { atoms treated by a mixture of } \\
\text { independent and constrained } \\
\text { refinement }\end{array}$ \\
\hline$\Delta \rho_{\max }, \Delta \rho_{\min }\left(\mathrm{e} \AA^{-3}\right)$ & $0.41,-0.44$ \\
\hline
\end{tabular}

Computer programs: APEX2 and SAINT (Bruker, 2013), SHELXT (Sheldrick, 2015a), SHELXL (Sheldrick, 2015b), ORTEP-3 for Windows (Farrugia, 2012) and PLATON (Spek, 2020).

123.5, 122.4, 94.7, 92.3, 81.2, 52.8, 48.8, 44.5, 39.7, 33.8, 21.0. MS (APCI): $m / z=453[M+\mathrm{H}]^{+}$.

\section{Refinement details}

Crystal data, data collection and structure refinement details are summarized in Table 4. The hydrogen atoms of the hydroxy groups were located in a difference-Fourier map and refined freely. The other hydrogen atoms were constrained to ride on their parent atoms with $\mathrm{C}-\mathrm{H}=0.95,0.98,0.99$ and $1.00 \AA$ for aromatic, methyl, methylene and methine $\mathrm{H}$ atoms, respectively. Isotropic displacement parameters of these atoms were constrained to $1.5 U_{\mathrm{eq}}(\mathrm{C})$ for the methyl and to $1.2 U_{\mathrm{eq}}(\mathrm{C})$ for all other $\mathrm{H}$ atoms.

\section{Funding information}

The authors are grateful to the Russian Foundation for Basic Research (RFBR) (award No. 19-53-04002, Bel_mol_a) and the Belarusian Republican Foundation for Fundamental Research (BRFFR) (award No. X19PM-003) for financial support of this research.

\section{References}

Afkhami, F. A., Khandar, A. A., Mahmoudi, G., Maniukiewicz, W., Gurbanov, A. V., Zubkov, F. I., Şahin, O., Yesilel, O. Z. \& Frontera, A. (2017). CrystEngComm, 19, 1389-1399.
Anderson, R. J., Groundwater, P. W., Todd, A. \& Worsley, A. J. (2012). Antibacterial Agents, ch. 3.1 Sulfonamide Antibacterial Agents, pp. 103-126. Chichester: John Wiley \& Sons, Ltd.

Asadov, Z. H., Rahimov, R. A., Ahmadova, G. A., Mammadova, K. A. \& Gurbanov, A. V. (2016). J. Surfact. Deterg. 19, 145-153.

Breytenbach, J. C., van Dyk, S., van den Heever, I., Allin, S. M., Hodkinson, C. C., Northfield, C. J. \& Page, M. I. (2000). Bioorg. Med. Chem. Lett. 10, 1629-1631.

Bruker (2013). APEX2, SAINT and SADABS. Bruker AXS Inc., Madison, Wisconsin, USA.

Cremer, D. \& Pople, J. A. (1975). J. Am. Chem. Soc. 97, 1354-1358.

Demircan, A., Kandemir, M. K., Colak, M. \& Karaarslan, M. (2016). Synthesis, 48, 2873-2880.

Demircan, A., Temel, E., Kandemir, M. K., Çolak, M. \& Büyükgüngör, O. (2013). Acta Cryst. E69, o1628-o1629.

Farrugia, L. J. (2012). J. Appl. Cryst. 45, 849-854.

Groom, C. R., Bruno, I. J., Lightfoot, M. P. \& Ward, S. C. (2016). Acta Cryst. B72, 171-179.

Gurbanov, A. V., Mahmoudi, G., Guedes da Silva, M. F. C., Zubkov, F. I., Mahmudov, K. T. \& Pombeiro, A. J. L. (2018). Inorg. Chim. Acta, 471, 130-136.

Gurbanov, A. V., Mahmudov, K. T., Sutradhar, M., Guedes da Silva, F. C., Mahmudov, T. A., Guseinov, F. I., Zubkov, F. I., Maharramov, A. M. \& Pombeiro, A. J. L. (2017). J. Organomet. Chem. 834, 22-27.

Karmakar, A., Rúbio, G. M. D. M., Paul, A., Guedes da Silva, M. F. C., Mahmudov, K. T., Guseinov, F. I., Carabineiro, S. A. C. \& Pombeiro, A. J. L. (2017). Dalton Trans. 46, 8649-8657.

Kopylovich, M. N., Mahmudov, K. T., Haukka, M., Luzyanin, K. V. \& Pombeiro, A. J. L. (2011a). Inorg. Chim. Acta, 374, 175-180.

Kopylovich, M. N., Mahmudov, K. T., Mizar, A. \& Pombeiro, A. J. L. (2011b). Chem. Commun. 47, 7248-7250.

Koşar, B., Demircan, A., Arslan, H. \& Büyükgüngör, O. (2011). Acta Cryst. E67, o994-0995.

Koşar, B., Karaarslan, M., Demir, I. \& Büyükgüngör, O. (2007). Acta Cryst. E63, o3323.

Ma, Z., Gurbanov, A. V., Maharramov, A. M., Guseinov, F. I., Kopylovich, M. N., Zubkov, F. I., Mahmudov, K. T. \& Pombeiro, A. J. L. (2017a). J. Mol. Catal. A Chem. 426, 526-533.

Ma, Z., Gurbanov, A. V., Sutradhar, M., Kopylovich, M. N., Mahmudov, K. T., Maharramov, A. M., Guseinov, F. I., Zubkov, F. I. \& Pombeiro, A. J. L. (2017b). Mol. Catal. 428, 17-23.

Maharramov, A. M., Shikhaliyev, N. Q., Suleymanova, G. T., Gurbanov, A. V., Babayeva, G. V., Mammadova, G. Z., Zubkov, F. I., Nenajdenko, V. G., Mahmudov, K. T. \& Pombeiro, A. J. L. (2018). Dyes Pigments, 159, 135-141.

Mahmoudi, G., Dey, L., Chowdhury, H., Bauzá, A., Ghosh, B. K., Kirillov, A. M., Seth, S. K., Gurbanov, A. V. \& Frontera, A. (2017). Inorg. Chim. Acta, 461, 192-205.

Mahmoudi, G., Khandar, A. A., Afkhami, F. A., Miroslaw, B., Gurbanov, A. V., Zubkov, F. I., Kennedy, A., Franconetti, A. \& Frontera, A. (2019). CrystEngComm, 21, 108-117.

Mahmudov, K. T., Gurbanov, A. V., Aliyeva, V. A., Resnati, G. \& Pombeiro, A. J. L. (2020). Coord. Chem. Rev. 418, 213381.

Mahmudov, K. T., Maharramov, A. M., Aliyeva, R. A., Aliyev, I. A., Kopylovich, M. N. \& Pombeiro, A. J. L. (2010). Anal. Lett. 43, 29232938.

Mitchell, L. A., Stanley, J. M., Espinosa De Hoyos, L. \& Holliday, B. J. (2013). Acta Cryst. C69, 638-641.

Mizar, A., Guedes da Silva, M. F. C., Kopylovich, M. N., Mukherjee, S., Mahmudov, K. T. \& Pombeiro, A. J. L. (2012). Eur. J. Inorg. Chem. pp. 2305-2313.

Nadirova, M. A., Khanova, A. V., Zubkov, F. I., Mertsalov, D. F., Kolesnik, I. A., Petkevich, S. K., Potkin, V. I., Shetnev, A. A., Presnukhina, S. I., Sinelshchikova, A. A., Grigoriev, M. S. \& Zaytsev, V. P. (2020). Tetrahedron. In the press.

Sheldrick, G. M. (2015a). Acta Cryst. A71, 3-8.

Sheldrick, G. M. (2015b). Acta Cryst. C71, 3-8.

Spek, A. L. (2020). Acta Cryst. E76, 1-11. 
Stiefl, N. \& Baumann, K. (2003). J. Med. Chem. 46, 1390-1407.

Sutradhar, M., Martins, L. M. D. R. S., Guedes da Silva, M. F. C., Mahmudov, K. T., Liu, C.-M. \& Pombeiro, A. J. L. (2015). Eur. J. Inorg. Chem. pp. 3959-3969.

Temel, E., Demircan, A., Arslan, H. \& Büyükgüngör, O. (2011). Acta Cryst. E67, o1304-o1305.

Temel, E., Demircan, A., Beyazova, G. \& Büyükgüngör, O. (2012). Acta Cryst. E68, o1102-o1103.

Temel, E., Demircan, A., Kandemir, M. K., Çolak, M. \& Büyükgüngör, O. (2013). Acta Cryst. E69, o1551-o1552.
Tietze, L. F. \& Beifuss, U. (1993). Angew. Chem. Int. Ed. Engl. 32, 131-163.

Turner, M. J., McKinnon, J. J., Wolff, S. K., Grimwood, D. J., Spackman, P. R., Jayatilaka, D. \& Spackman, M. A. (2017). CrystalExplorer17. The University of Western Australia.

Zubkov, F. I., Nikitina, E. V., Galeev, T. R., Zaytsev, V. P., Khrustalev, V. N., Novikov, R. A., Orlova, D. N. \& Varlamov, A. V. (2014). Tetrahedron, 70, 1659-1690.

Zubkov, F. I., Nikitina, E. V. \& Varlamov, A. V. (2005). Russ. Chem. Rev. 74, 639-669. 


\section{supporting information}

Acta Cryst. (2021). E77, 260-265 [https://doi.org/10.1107/S2056989021001626]

Crystal structure and Hirshfeld surface analysis of (RS)-3-hydroxy-2-\{[(3aRS,6RS,7aRS)-2-(4-methylphenylsulfonyl)-2,3,3a,6,7,7a-hexahydro-3a,6-epoxy-1H-isoindol-6-yl]methyl\}isoindolin-1-one

Dmitriy F. Mertsalov, Maryana A. Nadirova, Elena A. Sorokina, Marina A. Vinokurova, Sevim Türktekin Çelikesir, Mehmet Akkurt, Irina A. Kolesnik and Ajaya Bhattarai

Computing details

Data collection: APEX2 (Bruker, 2013); cell refinement: SAINT (Bruker, 2013); data reduction: SAINT (Bruker, 2013); program(s) used to solve structure: SHELXT (Sheldrick, 2015a); program(s) used to refine structure: SHELXL

(Sheldrick, 2015b); molecular graphics: ORTEP-3 for Windows (Farrugia, 2012); software used to prepare material for publication: PLATON (Spek, 2020).

(RS)-3-Hydroxy-2-\{[(3aRS,6RS,7aRS)-2-(4-methylphenylsulfonyl)-2,3,3a,6,7,7a-hexahydro-3a,6-epoxy-1Hisoindol-6-yl]methyl\}isoindolin-1-one

Crystal data

$\mathrm{C}_{24} \mathrm{H}_{24} \mathrm{~N}_{2} \mathrm{O}_{5} \mathrm{~S}$

$M_{r}=452.51$

Triclinic, $P \overline{1}$

$a=11.8210(8) \AA$

$b=11.8395(8) \AA$

$c=16.7336(11) \AA$

$\alpha=77.949(1)^{\circ}$

$\beta=79.555(1)^{\circ}$

$\gamma=77.511(1)^{\circ}$

$V=2213.3(3) \AA^{3}$

Data collection

Bruker APEXII CCD

diffractometer

Radiation source: sealed tube

Graphite monochromator

$\varphi$ and $\omega$ scans

Absorption correction: multi-scan

(SADABS; Bruker, 2013)

$T_{\min }=0.688, T_{\max }=0.746$

Refinement

Refinement on $F^{2}$

Least-squares matrix: full

$R\left[F^{2}>2 \sigma\left(F^{2}\right)\right]=0.060$
$Z=4$

$F(000)=952$

$D_{\mathrm{x}}=1.358 \mathrm{Mg} \mathrm{m}^{-3}$

Mo $K \alpha$ radiation, $\lambda=0.71073 \AA$

Cell parameters from 5394 reflections

$\theta=2.3-27.2^{\circ}$

$\mu=0.19 \mathrm{~mm}^{-1}$

$T=120 \mathrm{~K}$

Prism, colourless

$0.15 \times 0.09 \times 0.06 \mathrm{~mm}$

29692 measured reflections

13532 independent reflections

8799 reflections with $I>2 \sigma(I)$

$R_{\text {int }}=0.043$

$\theta_{\max }=30.6^{\circ}, \theta_{\min }=1.8^{\circ}$

$h=-16 \rightarrow 16$

$k=-16 \rightarrow 16$

$l=-23 \rightarrow 23$

$w R\left(F^{2}\right)=0.147$

$S=1.02$

13532 reflections 
581 parameters

0 restraints

Hydrogen site location: mixed

$\mathrm{H}$ atoms treated by a mixture of independent and constrained refinement

$$
\begin{aligned}
& w=1 /\left[\sigma^{2}\left(F_{\mathrm{o}}^{2}\right)+(0.0474 P)^{2}+1.6525 P\right] \\
& \text { where } P=\left(F_{\mathrm{o}}^{2}+2 F_{\mathrm{c}}^{2}\right) / 3 \\
& (\Delta / \sigma)_{\max }=0.001 \\
& \Delta \rho_{\max }=0.41 \mathrm{e} \AA^{-3} \\
& \Delta \rho_{\min }=-0.44 \mathrm{e} \AA^{-3}
\end{aligned}
$$

Special details

Geometry. All esds (except the esd in the dihedral angle between two 1.s. planes) are estimated using the full covariance

\begin{tabular}{|c|c|c|c|c|}
\hline & $x$ & $y$ & $z$ & $U_{\text {iso }} * / U_{\text {eq }}$ \\
\hline $\mathrm{S} 1$ & $0.74652(5)$ & $0.55414(5)$ & $0.53950(3)$ & $0.02594(13)$ \\
\hline $\mathrm{O} 1$ & $0.32628(13)$ & $0.11057(14)$ & $0.96738(9)$ & $0.0263(3)$ \\
\hline $\mathrm{O} 2$ & $0.71088(13)$ & $0.03280(14)$ & $0.88152(10)$ & 0.0278 \\
\hline $\mathrm{H} 2$ & 0.705699 & -0.016702 & 0.931497 & $0.042(8)^{*}$ \\
\hline $\mathrm{O} 3$ & $0.57170(13)$ & $0.31030(12)$ & $0.74923(9)$ & $0.0212(3)$ \\
\hline $\mathrm{O} 4$ & $0.75506(15)$ & $0.67311(14)$ & $0.53936(11)$ & $0.0353(4)$ \\
\hline O5 & $0.73338(14)$ & $0.51910(15)$ & $0.46520(10)$ & $0.0317(4)$ \\
\hline N1 & $0.50789(16)$ & $0.12179(15)$ & $0.89107(10)$ & $0.0221(4)$ \\
\hline N2 & $0.63120(16)$ & $0.52671(16)$ & $0.60548(11)$ & $0.0240(4)$ \\
\hline $\mathrm{C} 1$ & 0.41649 (19) & 0.06714 (19) & $0.92534(12)$ & $0.0216(4)$ \\
\hline $\mathrm{C} 2$ & 0.44704 (19) & $-0.05190(19)$ & 0.90475 (13) & 0.0235 \\
\hline $\mathrm{C} 3$ & $0.3823(2)$ & $-0.1414(2)$ & $0.92483(14)$ & $0.0280(5)$ \\
\hline H3 & 0.305319 & -0.130549 & 0.954375 & $0.034 *$ \\
\hline $\mathrm{C} 4$ & $0.4354(2)$ & $-0.2480(2)$ & $0.89974(15)$ & $0.0334(6)$ \\
\hline $\mathrm{H} 4$ & 0.393844 & -0.311270 & 0.912327 & $0.040 *$ \\
\hline $\mathrm{C} 5$ & $0.5482(2)$ & $-0.2629(2)$ & $0.85654(15)$ & $0.0339(6)$ \\
\hline H5 & 0.582760 & -0.336705 & 0.840882 & $0.041 *$ \\
\hline C6 & $0.6117(2)$ & $-0.1720(2)$ & $0.83572(15)$ & $0.0310(5)$ \\
\hline H6 & 0.688286 & -0.182161 & 0.805520 & $0.037 *$ \\
\hline $\mathrm{C} 7$ & $0.5589(2)$ & $-0.06640(19)$ & $0.86068(13)$ & $0.0242(4)$ \\
\hline $\mathrm{C} 8$ & 0.60631 (19) & $0.04558(19)$ & $0.84880(13)$ & $0.0245(4)$ \\
\hline H8 & 0.618437 & 0.079526 & 0.788610 & $0.029 *$ \\
\hline C9 & $0.5108(2)$ & 0.24198 (19) & $0.89628(13)$ & $0.0237(4)$ \\
\hline H9A & 0.589661 & 0.245866 & 0.906416 & $0.028 *$ \\
\hline H9B & 0.453931 & 0.264980 & 0.943998 & $0.028 *$ \\
\hline $\mathrm{C} 10$ & $0.48228(19)$ & $0.32883(18)$ & $0.81931(13)$ & $0.0217(4)$ \\
\hline $\mathrm{C} 11$ & $0.3729(2)$ & $0.3272(2)$ & $0.78461(14)$ & $0.0274(5)$ \\
\hline H11 & 0.304341 & 0.300502 & 0.814352 & $0.033 *$ \\
\hline $\mathrm{C} 12$ & $0.3917(2)$ & $0.3702(2)$ & $0.70465(14)$ & $0.0289(5)$ \\
\hline H12 & 0.340100 & 0.379979 & 0.665263 & $0.035^{*}$ \\
\hline C13 & 0.51173 (19) & 0.40013 (19) & $0.68953(13)$ & $0.0230(4)$ \\
\hline C14 & $0.5869(2)$ & $0.4180(2)$ & $0.60650(13)$ & $0.0266(5)$ \\
\hline H14A & 0.652259 & 0.350464 & 0.601447 & $0.032^{*}$ \\
\hline H14B & 0.540199 & 0.428583 & 0.561104 & $0.032 *$ \\
\hline
\end{tabular}
matrix. The cell esds are taken into account individually in the estimation of esds in distances, angles and torsion angles; correlations between esds in cell parameters are only used when they are defined by crystal symmetry. An approximate (isotropic) treatment of cell esds is used for estimating esds involving l.s. planes.

Fractional atomic coordinates and isotropic or equivalent isotropic displacement parameters $\left(\AA^{2}\right)$ 


\begin{tabular}{|c|c|c|c|c|}
\hline C15 & $0.4833(2)$ & $0.45976(19)$ & $0.82203(13)$ & $0.0271(5)$ \\
\hline H15A & 0.407907 & 0.498358 & 0.849741 & $0.032 *$ \\
\hline H15B & 0.547856 & 0.466503 & 0.850003 & $0.032 *$ \\
\hline C16 & $0.5028(2)$ & $0.51120(19)$ & $0.72882(13)$ & $0.0262(5)$ \\
\hline H16 & 0.434974 & 0.573493 & 0.712567 & $0.031 *$ \\
\hline $\mathrm{C} 17$ & $0.6189(2)$ & $0.5493(2)$ & $0.69070(14)$ & $0.0268(5)$ \\
\hline H17A & 0.615310 & 0.633446 & 0.691189 & $0.032 *$ \\
\hline H17B & 0.684024 & 0.501509 & 0.719696 & $0.032 *$ \\
\hline C18 & $0.86926(19)$ & $0.46288(19)$ & $0.57944(13)$ & $0.0237(4)$ \\
\hline C19 & $0.9263(2)$ & 0.5017 (2) & $0.63118(15)$ & $0.0295(5)$ \\
\hline H19 & 0.901762 & 0.579077 & 0.643101 & $0.035^{*}$ \\
\hline $\mathrm{C} 20$ & $1.0194(2)$ & $0.4264(2)$ & $0.66527(14)$ & $0.0298(5)$ \\
\hline $\mathrm{H} 20$ & 1.059570 & 0.453139 & 0.699867 & $0.036^{*}$ \\
\hline $\mathrm{C} 21$ & $1.0548(2)$ & $0.3122(2)$ & $0.64953(14)$ & $0.0268(5)$ \\
\hline $\mathrm{C} 22$ & $0.9972(2)$ & $0.2756(2)$ & $0.59696(14)$ & $0.0257(5)$ \\
\hline $\mathrm{H} 22$ & 1.021707 & 0.198304 & 0.584974 & $0.031 *$ \\
\hline $\mathrm{C} 23$ & $0.90444(19)$ & $0.3498(2)$ & $0.56163(13)$ & $0.0246(4)$ \\
\hline $\mathrm{H} 23$ & 0.865580 & 0.323813 & 0.525833 & $0.030 *$ \\
\hline $\mathrm{C} 24$ & $1.1564(2)$ & $0.2296(2)$ & $0.68509(17)$ & $0.0381(6)$ \\
\hline $\mathrm{H} 24 \mathrm{~A}$ & 1.136052 & 0.151744 & 0.705618 & $0.057^{*}$ \\
\hline H24B & 1.174711 & 0.259604 & 0.730684 & $0.057 *$ \\
\hline $\mathrm{H} 24 \mathrm{C}$ & 1.224817 & 0.223780 & 0.642151 & $0.057^{*}$ \\
\hline $\mathrm{S} 1^{\prime}$ & $0.76670(5)$ & $1.04803(5)$ & $0.53217(3)$ & $0.02277(12)$ \\
\hline $\mathrm{O} 1^{\prime}$ & $1.14505(15)$ & $0.50110(16)$ & $0.91923(11)$ & $0.0362(4)$ \\
\hline $\mathrm{O} 2^{\prime}$ & $0.76049(15)$ & $0.54898(17)$ & $0.93007(12)$ & $0.0410(5)$ \\
\hline $\mathrm{H} 2^{\prime}$ & 0.782753 & 0.534895 & 0.980589 & $0.078(12)^{*}$ \\
\hline O3' & $0.90933(13)$ & $0.76045(13)$ & $0.73711(9)$ & $0.0234(3)$ \\
\hline O4' & $0.80016(14)$ & $0.99924(15)$ & $0.45795(9)$ & $0.0300(4)$ \\
\hline O5' & $0.75650(14)$ & $1.17211(14)$ & $0.52883(10)$ & 0.0289 (4) \\
\hline $\mathrm{N} 1^{\prime}$ & 0.95957 (17) & $0.56602(18)$ & $0.88347(12)$ & $0.0302(4)$ \\
\hline $\mathrm{N} 2^{\prime}$ & $0.86625(15)$ & $0.98216(16)$ & $0.59042(11)$ & $0.0223(4)$ \\
\hline $\mathrm{C} 1^{\prime}$ & $1.0534(2)$ & $0.4821(2)$ & $0.90414(14)$ & $0.0297(5)$ \\
\hline $\mathrm{C} 2^{\prime}$ & $1.0214(2)$ & $0.3675(2)$ & $0.90349(14)$ & $0.0306(5)$ \\
\hline $\mathrm{C} 3^{\prime}$ & $1.0859(2)$ & $0.2544(2)$ & $0.91983(15)$ & $0.0349(6)$ \\
\hline H3' & 1.162486 & 0.240626 & 0.934224 & $0.042 *$ \\
\hline $\mathrm{C} 4^{\prime}$ & $1.0340(3)$ & $0.1626(3)$ & $0.91428(18)$ & $0.0469(7)$ \\
\hline $\mathrm{H} 4^{\prime}$ & 1.075607 & 0.084206 & 0.925324 & $0.056^{*}$ \\
\hline $\mathrm{C} 5^{\prime}$ & $0.9228(3)$ & $0.1828(3)$ & $0.8930(2)$ & $0.0513(8)$ \\
\hline $\mathrm{H} 5^{\prime}$ & 0.889731 & 0.118235 & 0.889034 & $0.062 *$ \\
\hline $\mathrm{C} 6^{\prime}$ & 0.8584 (3) & 0.2968 (3) & $0.87730(19)$ & $0.0457(7)$ \\
\hline $\mathrm{H} 6^{\prime}$ & 0.781783 & 0.310803 & 0.862951 & $0.055^{*}$ \\
\hline$C 7^{\prime}$ & $0.9096(2)$ & $0.3884(2)$ & $0.88328(15)$ & $0.0345(6)$ \\
\hline $\mathrm{C} 8^{\prime}$ & $0.8596(2)$ & $0.5185(2)$ & $0.87297(16)$ & $0.0350(6)$ \\
\hline $\mathrm{H} 8^{\prime}$ & 0.840457 & 0.547981 & 0.815582 & $0.042 *$ \\
\hline $\mathrm{C} 9^{\prime}$ & $0.9530(2)$ & $0.6906(2)$ & $0.87978(14)$ & $0.0314(5)$ \\
\hline $\mathrm{H}^{\prime} \mathrm{A}$ & 1.003483 & 0.701044 & 0.917835 & $0.038^{*}$ \\
\hline H9'B & 0.871471 & 0.726203 & 0.899287 & $0.038^{*}$ \\
\hline $\mathrm{C} 10^{\prime}$ & $0.98998(19)$ & $0.7553(2)$ & $0.79436(13)$ & 0.0239 \\
\hline
\end{tabular}




$\begin{array}{lllll}\text { C11' } & 1.1054(2) & 0.7027(2) & 0.74680(14) & 0.0260(5) \\ \text { H11' } & 1.170278 & 0.652311 & 0.769535 & 0.031^{*} \\ \text { C12' } & 1.09712(19) & 0.74141(19) & 0.66761(14) & 0.0251(5) \\ \text { H12' } & 1.154055 & 0.724037 & 0.621769 & 0.030^{*} \\ \text { C13' } & 0.97764(19) & 0.81916(19) & 0.66568(13) & 0.0221(4) \\ \text { C14' } & 0.9156(2) & 0.8553(2) & 0.59063(14) & 0.0264(5) \\ \text { H14C } & 0.971028 & 0.843925 & 0.539741 & 0.032^{*} \\ \text { H14D } & 0.852747 & 0.809951 & 0.595442 & 0.032^{*} \\ \text { C15' } & 0.9874(2) & 0.8886(2) & 0.78895(13) & 0.0255(5) \\ \text { H15C } & 0.918542 & 0.924993 & 0.824380 & 0.031^{*} \\ \text { H15D } & 1.059722 & 0.902498 & 0.804252 & 0.031^{*} \\ \text { C16' } & 0.97915(18) & 0.93444(19) & 0.69668(13) & 0.0223(4) \\ \text { H16' } & 1.048276 & 0.970275 & 0.668076 & 0.027^{*} \\ \text { C17' } & 0.86454(19) & 1.01119(19) & 0.67252(13) & 0.0222(4) \\ \text { H17C } & 0.796071 & 0.989347 & 0.711767 & 0.027^{*} \\ \text { H17D } & 0.864683 & 1.095695 & 0.668901 & 0.027^{*} \\ \text { C18' } & 0.63222(18) & 1.01004(19) & 0.58149(13) & 0.0226(4) \\ \text { C19' } & 0.6003(2) & 0.9103(2) & 0.56757(15) & 0.0285(5) \\ \text { H19' } & 0.648619 & 0.865397 & 0.529054 & 0.034^{*} \\ \text { C20' } & 0.4977(2) & 0.8770(2) & 0.61023(15) & 0.0308(5) \\ \text { H20' } & 0.476141 & 0.808453 & 0.601103 & 0.037^{*} \\ \text { C21' } & 0.4257(2) & 0.9424(2) & 0.66633(14) & 0.0293(5) \\ \text { C22' } & 0.4569(2) & 1.0438(2) & 0.67779(14) & 0.0283(5) \\ \text { H22' } & 0.407097 & 1.090152 & 0.714863 & 0.034^{*} \\ \text { C23' } & 0.55990(19) & 1.0784(2) & 0.63577(13) & 0.0248(4) \\ \text { H23' } & 0.580717 & 1.147840 & 0.643989 & 0.030^{*} \\ \text { C24' } & 0.3143(2) & 0.9045(3) & 0.71372(16) & 0.0400(6) \\ \text { H24D } & 0.285977 & 0.947062 & 0.759935 & 0.060^{*} \\ \text { H24E } & 0.330163 & 0.819809 & 0.734947 & 0.060^{*} \\ \text { H24F } & 0.254611 & 0.922022 & 0.676926 & 0.060^{*}\end{array}$

Atomic displacement parameters $\left(\AA^{2}\right)$

\begin{tabular}{lllllll}
\hline & $U^{11}$ & $U^{22}$ & $U^{33}$ & $U^{12}$ & $U^{13}$ & $U^{23}$ \\
\hline S1 & $0.0286(3)$ & $0.0229(3)$ & $0.0220(3)$ & $-0.0052(2)$ & $0.0008(2)$ & $0.0024(2)$ \\
O1 & $0.0220(8)$ & $0.0272(8)$ & $0.0249(8)$ & $-0.0003(6)$ & $-0.0020(6)$ & $0.0004(6)$ \\
O2 & $0.0233(8)$ & $0.0326(9)$ & $0.0245(8)$ & $-0.0072(7)$ & $0.0000(6)$ & $0.0010(7)$ \\
O3 & $0.0247(7)$ & $0.0199(7)$ & $0.0166(7)$ & $-0.0036(6)$ & $-0.0020(6)$ & $0.0007(5)$ \\
O4 & $0.0367(10)$ & $0.0216(8)$ & $0.0409(10)$ & $-0.0069(7)$ & $0.0025(8)$ & $0.0042(7)$ \\
O5 & $0.0322(9)$ & $0.0389(10)$ & $0.0194(8)$ & $-0.0058(7)$ & $-0.0017(7)$ & $0.0023(7)$ \\
N1 & $0.0260(9)$ & $0.0207(9)$ & $0.0175(8)$ & $-0.0043(7)$ & $-0.0010(7)$ & $-0.0003(7)$ \\
N2 & $0.0269(9)$ & $0.0236(9)$ & $0.0188(9)$ & $-0.0063(7)$ & $0.0026(7)$ & $-0.0008(7)$ \\
C1 & $0.0233(10)$ & $0.0237(11)$ & $0.0159(9)$ & $-0.0033(8)$ & $-0.0067(8)$ & $0.0035(8)$ \\
C2 & $0.0278(11)$ & $0.0228(11)$ & $0.0191(10)$ & $-0.0045(9)$ & $-0.0076(8)$ & $0.0015(8)$ \\
C3 & $0.0327(12)$ & $0.0272(12)$ & $0.0253(11)$ & $-0.0083(10)$ & $-0.0102(10)$ & $0.0010(9)$ \\
C4 & $0.0486(15)$ & $0.0247(12)$ & $0.0315(12)$ & $-0.0112(11)$ & $-0.0190(11)$ & $0.0005(10)$ \\
C5 & $0.0481(15)$ & $0.0242(12)$ & $0.0308(12)$ & $-0.0012(11)$ & $-0.0153(11)$ & $-0.0056(10)$ \\
C6 & $0.0355(13)$ & $0.0287(13)$ & $0.0266(12)$ & $0.0005(10)$ & $-0.0074(10)$ & $-0.0043(9)$
\end{tabular}




\begin{tabular}{|c|c|c|c|c|c|c|}
\hline $\mathrm{C} 7$ & $0.0291(11)$ & $0.0249(11)$ & $0.0170(10)$ & $-0.0030(9)$ & $-0.0047(8)$ & $-0.0013(8)$ \\
\hline $\mathrm{C} 8$ & $0.0254(11)$ & $0.0266(11)$ & $0.0179(10)$ & $-0.0025(9)$ & $0.0002(8)$ & $-0.0013(8)$ \\
\hline C9 & $0.0294(11)$ & $0.0218(11)$ & $0.0191(10)$ & $-0.0056(9)$ & $-0.0030(9)$ & $-0.0012(8)$ \\
\hline $\mathrm{C} 10$ & $0.0252(10)$ & $0.0208(10)$ & $0.0176(9)$ & $-0.0045(8)$ & $-0.0009(8)$ & $-0.0015(8)$ \\
\hline C11 & $0.0244(11)$ & $0.0292(12)$ & $0.0262(11)$ & $-0.0067(9)$ & $-0.0030(9)$ & $0.0018(9)$ \\
\hline C12 & $0.0265(11)$ & $0.0341(13)$ & $0.0247(11)$ & $-0.0072(10)$ & $-0.0071(9)$ & $0.0027(9)$ \\
\hline $\mathrm{C} 13$ & $0.0280(11)$ & $0.0201(10)$ & $0.0181(10)$ & $-0.0034(8)$ & $-0.0033(8)$ & $0.0018(8)$ \\
\hline $\mathrm{C} 14$ & $0.0342(12)$ & $0.0264(12)$ & $0.0195(10)$ & $-0.0110(9)$ & $-0.0011(9)$ & $-0.0015(8)$ \\
\hline $\mathrm{C} 15$ & $0.0360(13)$ & $0.0226(11)$ & $0.0203(10)$ & $-0.0070(9)$ & $0.0042(9)$ & $-0.0042(8)$ \\
\hline C16 & $0.0315(12)$ & $0.0203(11)$ & $0.0219(10)$ & $-0.0025(9)$ & $0.0005(9)$ & $0.0010(8)$ \\
\hline $\mathrm{C} 17$ & $0.0348(12)$ & $0.0205(11)$ & $0.0231(11)$ & $-0.0080(9)$ & $0.0015(9)$ & $-0.0016(8)$ \\
\hline C18 & $0.0260(11)$ & $0.0236(11)$ & $0.0203(10)$ & $-0.0068(9)$ & $0.0008(8)$ & $-0.0027(8)$ \\
\hline C19 & $0.0336(12)$ & $0.0250(12)$ & $0.0320(12)$ & $-0.0099(10)$ & $-0.0008(10)$ & $-0.0082(9)$ \\
\hline $\mathrm{C} 20$ & $0.0343(13)$ & $0.0346(13)$ & $0.0267(11)$ & $-0.0146(10)$ & $-0.0075(10)$ & $-0.0081(10)$ \\
\hline $\mathrm{C} 21$ & $0.0258(11)$ & $0.0324(13)$ & $0.0220(11)$ & $-0.0098(9)$ & -0.0007 (9) & $-0.0021(9)$ \\
\hline $\mathrm{C} 22$ & $0.0282(11)$ & $0.0237(11)$ & $0.0252(11)$ & $-0.0052(9)$ & $-0.0006(9)$ & $-0.0063(9)$ \\
\hline $\mathrm{C} 23$ & $0.0261(11)$ & $0.0269(12)$ & $0.0236(11)$ & $-0.0071(9)$ & $-0.0045(9)$ & $-0.0074(9)$ \\
\hline $\mathrm{C} 24$ & $0.0322(13)$ & $0.0454(16)$ & $0.0372(14)$ & $-0.0042(11)$ & $-0.0143(11)$ & $-0.0033(12)$ \\
\hline $\mathrm{S} 1^{\prime}$ & $0.0233(3)$ & $0.0244(3)$ & $0.0192(2)$ & $-0.0058(2)$ & $-0.0031(2)$ & $0.0010(2)$ \\
\hline $\mathrm{O} 1^{\prime}$ & $0.0288(9)$ & $0.0474(11)$ & $0.0299(9)$ & $-0.0071(8)$ & $-0.0058(7)$ & $0.0000(8)$ \\
\hline $\mathrm{O} 2^{\prime}$ & $0.0231(9)$ & $0.0504(12)$ & 0.0405 & -0.0009 (8) & $-0.0048(8)$ & $0.0061(8)$ \\
\hline O3' & $0.0216(7)$ & $0.0276(8)$ & $0.0200(7)$ & $-0.0079(6)$ & $-0.0011(6)$ & $-0.0002(6)$ \\
\hline O4' & $0.0309(9)$ & $0.0383(10)$ & $0.0192(8)$ & $-0.0050(7)$ & $-0.0038(7)$ & $-0.0029(7)$ \\
\hline O5' & $0.0312(9)$ & $0.0232(8)$ & $0.0311(9)$ & $-0.0088(7)$ & $-0.0046(7)$ & $0.0023(6)$ \\
\hline $\mathrm{N} 1^{\prime}$ & $0.0264(10)$ & $0.0315(11)$ & $0.0270(10)$ & $-0.0065(8)$ & $-0.0029(8)$ & $0.0076(8)$ \\
\hline $\mathrm{N} 2^{\prime}$ & $0.0214(9)$ & $0.0258(10)$ & $0.0188(8)$ & $-0.0025(7)$ & $-0.0037(7)$ & $-0.0031(7)$ \\
\hline $\mathrm{C}^{\prime \prime}$ & $0.0250(11)$ & 0.0389 (14) & $0.0186(10)$ & $-0.0036(10)$ & $0.0003(9)$ & $0.0041(9)$ \\
\hline $\mathrm{C} 2^{\prime}$ & $0.0254(11)$ & $0.0362(14)$ & $0.0245(11)$ & $-0.0044(10)$ & $0.0001(9)$ & $0.0031(10)$ \\
\hline C3' & $0.0320(13)$ & $0.0372(14)$ & $0.0260(12)$ & $-0.0002(11)$ & $0.0043(10)$ & $0.0019(10)$ \\
\hline $\mathrm{C} 4^{\prime}$ & $0.0482(17)$ & $0.0344(15)$ & 0.0467 (17) & $-0.0011(13)$ & $0.0068(14)$ & $-0.0007(12)$ \\
\hline $\mathrm{C} 5^{\prime}$ & $0.0491(18)$ & $0.0444(18)$ & $0.060(2)$ & $-0.0168(14)$ & $0.0031(15)$ & $-0.0084(15)$ \\
\hline $\mathrm{C} 6^{\prime}$ & $0.0356(15)$ & 0.0487 (18) & $0.0520(17)$ & $-0.0157(13)$ & $-0.0054(13)$ & $0.0007(14)$ \\
\hline$C 7^{\prime}$ & $0.0292(12)$ & $0.0395(15)$ & $0.0292(12)$ & $-0.0070(11)$ & $-0.0008(10)$ & $0.0046(10)$ \\
\hline $\mathrm{C}^{\prime}$ & $0.0248(12)$ & $0.0422(15)$ & $0.0323(13)$ & $-0.0055(10)$ & $-0.0053(10)$ & $0.0067(11)$ \\
\hline $\mathrm{C} 9^{\prime}$ & $0.0330(13)$ & $0.0342(13)$ & $0.0224(11)$ & $-0.0049(10)$ & $-0.0003(10)$ & $0.0009(9)$ \\
\hline $\mathrm{C} 10^{\prime}$ & $0.0222(10)$ & $0.0295(12)$ & $0.0185(10)$ & $-0.0059(9)$ & $-0.0027(8)$ & $0.0000(8)$ \\
\hline $\mathrm{C} 11^{\prime}$ & $0.0236(11)$ & $0.0256(11)$ & $0.0267(11)$ & $-0.0035(9)$ & $-0.0027(9)$ & $-0.0017(9)$ \\
\hline $\mathrm{C} 12^{\prime}$ & $0.0227(11)$ & $0.0254(11)$ & $0.0254(11)$ & $-0.0030(9)$ & $-0.0006(9)$ & $-0.0044(9)$ \\
\hline C13' & $0.0235(10)$ & $0.0245(11)$ & $0.0178(10)$ & $-0.0066(8)$ & $-0.0005(8)$ & $-0.0025(8)$ \\
\hline C14' & $0.0311(12)$ & $0.0262(12)$ & $0.0228(11)$ & $-0.0009(9)$ & $-0.0070(9)$ & $-0.0080(9)$ \\
\hline $\mathrm{C} 15^{\prime}$ & $0.0261(11)$ & $0.0321(12)$ & $0.0198(10)$ & $-0.0067(9)$ & $-0.0049(9)$ & $-0.0051(9)$ \\
\hline $\mathrm{C} 16^{\prime}$ & $0.0214(10)$ & $0.0252(11)$ & $0.0209(10)$ & $-0.0074(8)$ & $-0.0025(8)$ & $-0.0026(8)$ \\
\hline $\mathrm{C} 17^{\prime}$ & $0.0239(10)$ & $0.0227(11)$ & $0.0200(10)$ & $-0.0057(8)$ & $-0.0015(8)$ & $-0.0041(8)$ \\
\hline $\mathrm{C} 18^{\prime}$ & $0.0201(10)$ & $0.0235(11)$ & $0.0226(10)$ & $-0.0039(8)$ & $-0.0058(8)$ & $0.0017(8)$ \\
\hline C19' & $0.0285(12)$ & $0.0235(11)$ & $0.0342(12)$ & $-0.0032(9)$ & $-0.0086(10)$ & $-0.0053(9)$ \\
\hline $\mathrm{C} 20^{\prime}$ & $0.0305(12)$ & $0.0259(12)$ & 0.0389 (14) & $-0.0104(10)$ & $-0.0138(10)$ & $0.0009(10)$ \\
\hline $\mathrm{C} 21^{\prime}$ & $0.0239(11)$ & $0.0348(13)$ & $0.0276(12)$ & $-0.0092(9)$ & $-0.0134(9)$ & $0.0103(10)$ \\
\hline $\mathrm{C} 22^{\prime}$ & $0.0234(11)$ & $0.0358(13)$ & $0.0236(11)$ & $-0.0022(9)$ & $-0.0055(9)$ & $-0.0021(9)$ \\
\hline
\end{tabular}




$\begin{array}{lllllll}\mathrm{C} 23^{\prime} & 0.0248(11) & 0.0257(11) & 0.0239(11) & -0.0057(9) & -0.0051(9) & -0.0021(8) \\ \mathrm{C} 24^{\prime} & 0.0281(13) & 0.0547(17) & 0.0358(14) & -0.0165(12) & -0.0121(11) & 0.0109(12)\end{array}$

Geometric parameters $\left(A,{ }^{\circ}\right)$

\begin{tabular}{|c|c|c|c|}
\hline $\mathrm{S} 1-\mathrm{O} 4$ & $1.4332(17)$ & $\mathrm{S} 1^{\prime}-\mathrm{O} 4^{\prime}$ & $1.4337(17)$ \\
\hline $\mathrm{S} 1-\mathrm{O} 5$ & $1.4337(17)$ & $\mathrm{S} 1^{\prime}-\mathrm{O} 5^{\prime}$ & $1.4378(17)$ \\
\hline $\mathrm{S} 1-\mathrm{N} 2$ & $1.6368(19)$ & $\mathrm{S} 1^{\prime}-\mathrm{N} 2^{\prime}$ & $1.6263(18)$ \\
\hline $\mathrm{S} 1-\mathrm{C} 18$ & $1.758(2)$ & $\mathrm{S} 11^{\prime}-\mathrm{C} 18^{\prime}$ & $1.758(2)$ \\
\hline $\mathrm{O} 1-\mathrm{C} 1$ & $1.240(3)$ & $\mathrm{O} 1^{\prime}-\mathrm{C} 1^{\prime}$ & $1.231(3)$ \\
\hline $\mathrm{O} 2-\mathrm{C} 8$ & $1.407(3)$ & $\mathrm{O} 2^{\prime}-\mathrm{C} 8^{\prime}$ & $1.404(3)$ \\
\hline $\mathrm{O} 2-\mathrm{H} 2$ & 0.9160 & $\mathrm{O} 2^{\prime}-\mathrm{H} 2^{\prime}$ & 0.9026 \\
\hline $\mathrm{O} 3-\mathrm{C} 10$ & $1.449(2)$ & $\mathrm{O}^{\prime}-\mathrm{C} 13^{\prime}$ & $1.452(2)$ \\
\hline $\mathrm{O} 3-\mathrm{C} 13$ & $1.456(2)$ & $\mathrm{O}^{\prime}{ }^{\prime}-\mathrm{C} 10^{\prime}$ & $1.453(3)$ \\
\hline $\mathrm{N} 1-\mathrm{C} 1$ & $1.356(3)$ & $\mathrm{N} 1^{\prime}-\mathrm{C} 1^{\prime}$ & $1.363(3)$ \\
\hline $\mathrm{N} 1-\mathrm{C} 9$ & $1.452(3)$ & $\mathrm{N} 1^{\prime}-\mathrm{C} 9^{\prime}$ & $1.449(3)$ \\
\hline $\mathrm{N} 1-\mathrm{C} 8$ & $1.477(3)$ & $\mathrm{N} 1^{\prime}-\mathrm{C} 8^{\prime}$ & $1.466(3)$ \\
\hline $\mathrm{N} 2-\mathrm{C} 17$ & $1.481(3)$ & $\mathrm{N} 2^{\prime}-\mathrm{C} 17^{\prime}$ & $1.480(3)$ \\
\hline $\mathrm{N} 2-\mathrm{C} 14$ & $1.487(3)$ & $\mathrm{N} 2^{\prime}-\mathrm{C} 14^{\prime}$ & $1.488(3)$ \\
\hline $\mathrm{C} 1-\mathrm{C} 2$ & $1.474(3)$ & $\mathrm{C} 1^{\prime}-\mathrm{C} 2^{\prime}$ & $1.488(4)$ \\
\hline $\mathrm{C} 2-\mathrm{C} 7$ & $1.387(3)$ & $\mathrm{C} 2^{\prime}-\mathrm{C} 7^{\prime}$ & $1.382(3)$ \\
\hline $\mathrm{C} 2-\mathrm{C} 3$ & $1.390(3)$ & $\mathrm{C} 2^{\prime}-\mathrm{C} 3^{\prime}$ & $1.389(3)$ \\
\hline $\mathrm{C} 3-\mathrm{C} 4$ & $1.394(3)$ & $\mathrm{C} 3^{\prime}-\mathrm{C} 4^{\prime}$ & $1.385(4)$ \\
\hline $\mathrm{C} 3-\mathrm{H} 3$ & 0.9500 & $\mathrm{C}^{\prime}-\mathrm{H} 3^{\prime}$ & 0.9500 \\
\hline $\mathrm{C} 4-\mathrm{C} 5$ & $1.391(4)$ & $\mathrm{C} 4^{\prime}-\mathrm{C} 5^{\prime}$ & $1.381(4)$ \\
\hline $\mathrm{C} 4-\mathrm{H} 4$ & 0.9500 & $\mathrm{C} 4^{\prime}-\mathrm{H} 4^{\prime}$ & 0.9500 \\
\hline $\mathrm{C} 5-\mathrm{C} 6$ & $1.393(4)$ & $\mathrm{C}^{\prime}-\mathrm{C} 6^{\prime}$ & $1.396(4)$ \\
\hline $\mathrm{C} 5-\mathrm{H} 5$ & 0.9500 & $\mathrm{C}^{\prime}-\mathrm{H} 5^{\prime}$ & 0.9500 \\
\hline $\mathrm{C} 6-\mathrm{C} 7$ & $1.383(3)$ & $\mathrm{C} 6^{\prime}-\mathrm{C} 7^{\prime}$ & $1.379(4)$ \\
\hline C6-H6 & 0.9500 & $\mathrm{C} 6^{\prime}-\mathrm{H} 6^{\prime}$ & 0.9500 \\
\hline $\mathrm{C} 7-\mathrm{C} 8$ & $1.511(3)$ & $\mathrm{C} 7^{\prime}-\mathrm{C} 8^{\prime}$ & $1.511(4)$ \\
\hline $\mathrm{C} 8-\mathrm{H} 8$ & 1.0000 & $\mathrm{C} 8^{\prime}-\mathrm{H} 8^{\prime}$ & 1.0000 \\
\hline $\mathrm{C} 9-\mathrm{C} 10$ & $1.511(3)$ & $\mathrm{C} 9^{\prime}-\mathrm{C} 10^{\prime}$ & $1.510(3)$ \\
\hline C9- $\mathrm{H} 9 \mathrm{~A}$ & 0.9900 & $\mathrm{C} 9^{\prime}-\mathrm{H} 9^{\prime} \mathrm{A}$ & 0.9900 \\
\hline С9- & 0.9900 & $\mathrm{C} 9^{\prime}-\mathrm{H} 9^{\prime} \mathrm{B}$ & 0.9900 \\
\hline $\mathrm{C} 10-\mathrm{C} 11$ & $1.516(3)$ & $\mathrm{C} 10^{\prime}-\mathrm{C} 11^{\prime}$ & $1.526(3)$ \\
\hline $\mathrm{C} 10-\mathrm{C} 15$ & $1.563(3)$ & $\mathrm{C} 10^{\prime}-\mathrm{C} 15^{\prime}$ & $1.557(3)$ \\
\hline $\mathrm{C} 11-\mathrm{C} 12$ & $1.325(3)$ & $\mathrm{C} 11^{\prime}-\mathrm{C} 12^{\prime}$ & $1.323(3)$ \\
\hline $\mathrm{C} 11-\mathrm{H} 11$ & 0.9500 & $\mathrm{C} 11^{\prime}-\mathrm{H} 11^{\prime}$ & 0.9500 \\
\hline $\mathrm{C} 12-\mathrm{C} 13$ & $1.502(3)$ & $\mathrm{C} 12^{\prime}-\mathrm{C} 13^{\prime}$ & $1.512(3)$ \\
\hline $\mathrm{C} 12-\mathrm{H} 12$ & 0.9500 & $\mathrm{C} 12^{\prime}-\mathrm{H} 12^{\prime}$ & 0.9500 \\
\hline $\mathrm{C} 13-\mathrm{C} 14$ & $1.509(3)$ & $\mathrm{C} 13^{\prime}-\mathrm{C} 14^{\prime}$ & $1.507(3)$ \\
\hline $\mathrm{C} 13-\mathrm{C} 16$ & $1.565(3)$ & $\mathrm{C} 13^{\prime}-\mathrm{C} 16^{\prime}$ & $1.563(3)$ \\
\hline $\mathrm{C} 14-\mathrm{H} 14 \mathrm{~A}$ & 0.9900 & $\mathrm{C} 14^{\prime}-\mathrm{H} 14 \mathrm{C}$ & 0.9900 \\
\hline C14-H14B & 0.9900 & C14'-H14D & 0.9900 \\
\hline $\mathrm{C} 15-\mathrm{C} 16$ & $1.546(3)$ & $\mathrm{C} 15^{\prime}-\mathrm{C} 16^{\prime}$ & $1.540(3)$ \\
\hline C15-H15A & 0.9900 & $\mathrm{C} 15^{\prime}-\mathrm{H} 15 \mathrm{C}$ & 0.9900 \\
\hline $\mathrm{C} 15-\mathrm{H} 15 \mathrm{~B}$ & 0.9900 & $\mathrm{C} 15^{\prime}-\mathrm{H} 15 \mathrm{D}$ & 0.9900 \\
\hline
\end{tabular}




\begin{tabular}{|c|c|c|c|}
\hline $\mathrm{C} 16-\mathrm{C} 17$ & $1.525(3)$ & $\mathrm{C} 16^{\prime}-\mathrm{C} 17^{\prime}$ & $1.529(3)$ \\
\hline $\mathrm{C} 16-\mathrm{H} 16$ & 1.0000 & $\mathrm{C} 16^{\prime}-\mathrm{H} 16^{\prime}$ & 1.0000 \\
\hline C17-H17A & 0.9900 & $\mathrm{C} 17^{\prime}-\mathrm{H} 17 \mathrm{C}$ & 0.9900 \\
\hline C17-H17B & 0.9900 & C17'-H17D & 0.9900 \\
\hline $\mathrm{C} 18-\mathrm{C} 19$ & $1.388(3)$ & $\mathrm{C} 18^{\prime}-\mathrm{C} 19^{\prime}$ & $1.389(3)$ \\
\hline $\mathrm{C} 18-\mathrm{C} 23$ & $1.391(3)$ & $\mathrm{C} 18^{\prime}-\mathrm{C} 23^{\prime}$ & $1.392(3)$ \\
\hline $\mathrm{C} 19-\mathrm{C} 20$ & $1.387(3)$ & $\mathrm{C} 19^{\prime}-\mathrm{C} 20^{\prime}$ & $1.383(3)$ \\
\hline C19-H19 & 0.9500 & $\mathrm{C} 19^{\prime}-\mathrm{H} 19^{\prime}$ & 0.9500 \\
\hline $\mathrm{C} 20-\mathrm{C} 21$ & $1.392(3)$ & $\mathrm{C} 20^{\prime}-\mathrm{C} 21^{\prime}$ & $1.391(4)$ \\
\hline $\mathrm{C} 20-\mathrm{H} 20$ & 0.9500 & $\mathrm{C} 20^{\prime}-\mathrm{H} 20^{\prime}$ & 0.9500 \\
\hline $\mathrm{C} 21-\mathrm{C} 22$ & $1.389(3)$ & $\mathrm{C} 21^{\prime}-\mathrm{C} 22^{\prime}$ & $1.388(3)$ \\
\hline $\mathrm{C} 21-\mathrm{C} 24$ & $1.504(3)$ & $\mathrm{C} 21^{\prime}-\mathrm{C} 24^{\prime}$ & $1.515(3)$ \\
\hline $\mathrm{C} 22-\mathrm{C} 23$ & $1.387(3)$ & $\mathrm{C} 22^{\prime}-\mathrm{C} 23^{\prime}$ & $1.389(3)$ \\
\hline $\mathrm{C} 22-\mathrm{H} 22$ & 0.9500 & $\mathrm{C} 22^{\prime}-\mathrm{H} 22^{\prime}$ & 0.9500 \\
\hline $\mathrm{C} 23-\mathrm{H} 23$ & 0.9500 & $\mathrm{C} 23^{\prime}-\mathrm{H} 23^{\prime}$ & 0.9500 \\
\hline $\mathrm{C} 24-\mathrm{H} 24 \mathrm{~A}$ & 0.9800 & $\mathrm{C} 24^{\prime}-\mathrm{H} 24 \mathrm{D}$ & 0.9800 \\
\hline $\mathrm{C} 24-\mathrm{H} 24 \mathrm{~B}$ & 0.9800 & $\mathrm{C} 24^{\prime}-\mathrm{H} 24 \mathrm{E}$ & 0.9800 \\
\hline $\mathrm{C} 24-\mathrm{H} 24 \mathrm{C}$ & 0.9800 & $\mathrm{C} 24^{\prime}-\mathrm{H} 24 \mathrm{~F}$ & 0.9800 \\
\hline $\mathrm{O} 4-\mathrm{S} 1-\mathrm{O} 5$ & $120.45(10)$ & $\mathrm{O} 4^{\prime}-\mathrm{S} 1^{\prime}-\mathrm{O} 5^{\prime}$ & $120.20(10)$ \\
\hline $\mathrm{O} 4-\mathrm{S} 1-\mathrm{N} 2$ & $106.71(10)$ & $\mathrm{O} 4^{\prime}-\mathrm{S} 1^{\prime}-\mathrm{N} 2^{\prime}$ & $105.39(10)$ \\
\hline $\mathrm{O} 5-\mathrm{S} 1-\mathrm{N} 2$ & $105.53(10)$ & $\mathrm{O} 5^{\prime}-\mathrm{S} 1^{\prime}-\mathrm{N} 2^{\prime}$ & $106.73(10)$ \\
\hline $\mathrm{O} 4-\mathrm{S} 1-\mathrm{C} 18$ & $107.32(11)$ & $\mathrm{O} 4^{\prime}-\mathrm{S} 1^{\prime}-\mathrm{C} 18^{\prime}$ & $108.51(10)$ \\
\hline $\mathrm{O} 5-\mathrm{S} 1-\mathrm{C} 18$ & $109.11(10)$ & $\mathrm{O} 5^{\prime}-\mathrm{S} 1^{\prime}-\mathrm{C} 18^{\prime}$ & $107.32(10)$ \\
\hline $\mathrm{N} 2-\mathrm{S} 1-\mathrm{C} 18$ & $107.01(10)$ & $\mathrm{N} 2^{\prime}-\mathrm{S} 1^{\prime}-\mathrm{C} 18^{\prime}$ & $108.20(10)$ \\
\hline $\mathrm{C} 8-\mathrm{O} 2-\mathrm{H} 2$ & 108.3 & $\mathrm{C} 8^{\prime}-\mathrm{O} 2^{\prime}-\mathrm{H} 2^{\prime}$ & 108.3 \\
\hline $\mathrm{C} 10-\mathrm{O} 3-\mathrm{C} 13$ & $95.72(15)$ & $\mathrm{C} 13^{\prime}-\mathrm{O} 3^{\prime}-\mathrm{C} 10^{\prime}$ & 95.37 (15) \\
\hline $\mathrm{C} 1-\mathrm{N} 1-\mathrm{C} 9$ & $123.97(18)$ & $\mathrm{C} 1^{\prime}-\mathrm{N} 1^{\prime}-\mathrm{C} 9^{\prime}$ & $123.9(2)$ \\
\hline $\mathrm{C} 1-\mathrm{N} 1-\mathrm{C} 8$ & $113.04(18)$ & $\mathrm{C} 1^{\prime}-\mathrm{N} 1^{\prime}-\mathrm{C} 8^{\prime}$ & $113.8(2)$ \\
\hline $\mathrm{C} 9-\mathrm{N} 1-\mathrm{C} 8$ & $122.98(17)$ & $\mathrm{C} 9^{\prime}-\mathrm{N} 1^{\prime}-\mathrm{C} 8^{\prime}$ & $122.0(2)$ \\
\hline $\mathrm{C} 17-\mathrm{N} 2-\mathrm{C} 14$ & $109.96(16)$ & $\mathrm{C} 17^{\prime}-\mathrm{N} 2^{\prime}-\mathrm{C} 14^{\prime}$ & $110.14(16)$ \\
\hline $\mathrm{C} 17-\mathrm{N} 2-\mathrm{S} 1$ & $119.69(15)$ & $\mathrm{C} 17^{\prime}-\mathrm{N} 2^{\prime}-\mathrm{S} 1^{\prime}$ & $121.96(14)$ \\
\hline $\mathrm{C} 14-\mathrm{N} 2-\mathrm{S} 1$ & $118.46(14)$ & $\mathrm{C} 14^{\prime}-\mathrm{N} 2^{\prime}-\mathrm{S} 1^{\prime}$ & $119.12(14)$ \\
\hline $\mathrm{O} 1-\mathrm{C} 1-\mathrm{N} 1$ & $125.2(2)$ & $\mathrm{O} 1^{\prime}-\mathrm{C} 1^{\prime}-\mathrm{N} 1^{\prime}$ & $125.3(2)$ \\
\hline $\mathrm{O} 1-\mathrm{C} 1-\mathrm{C} 2$ & $127.7(2)$ & $\mathrm{O} 1^{\prime}-\mathrm{C} 1^{\prime}-\mathrm{C} 2^{\prime}$ & $128.7(2)$ \\
\hline $\mathrm{N} 1-\mathrm{C} 1-\mathrm{C} 2$ & $107.04(18)$ & $\mathrm{N} 1^{\prime}-\mathrm{C} 1^{\prime}-\mathrm{C} 2^{\prime}$ & $106.0(2)$ \\
\hline $\mathrm{C} 7-\mathrm{C} 2-\mathrm{C} 3$ & $121.9(2)$ & $\mathrm{C} 7^{\prime}-\mathrm{C} 2^{\prime}-\mathrm{C} 3^{\prime}$ & $121.8(2)$ \\
\hline $\mathrm{C} 7-\mathrm{C} 2-\mathrm{C} 1$ & $108.71(19)$ & $\mathrm{C} 7^{\prime}-\mathrm{C} 2^{\prime}-\mathrm{C} 1^{\prime}$ & $108.7(2)$ \\
\hline $\mathrm{C} 3-\mathrm{C} 2-\mathrm{C} 1$ & $129.4(2)$ & $\mathrm{C} 3^{\prime}-\mathrm{C} 2^{\prime}-\mathrm{C} 1^{\prime}$ & $129.6(2)$ \\
\hline $\mathrm{C} 2-\mathrm{C} 3-\mathrm{C} 4$ & $117.1(2)$ & $\mathrm{C} 4^{\prime}-\mathrm{C} 3^{\prime}-\mathrm{C} 2^{\prime}$ & $117.2(3)$ \\
\hline $\mathrm{C} 2-\mathrm{C} 3-\mathrm{H} 3$ & 121.5 & $\mathrm{C} 4^{\prime}-\mathrm{C} 3^{\prime}-\mathrm{H} 3^{\prime}$ & 121.4 \\
\hline $\mathrm{C} 4-\mathrm{C} 3-\mathrm{H} 3$ & 121.5 & $\mathrm{C} 2^{\prime}-\mathrm{C} 3^{\prime}-\mathrm{H} 3^{\prime}$ & 121.4 \\
\hline $\mathrm{C} 5-\mathrm{C} 4-\mathrm{C} 3$ & $120.9(2)$ & $\mathrm{C} 5^{\prime}-\mathrm{C} 4^{\prime}-\mathrm{C} 3^{\prime}$ & $121.3(3)$ \\
\hline $\mathrm{C} 5-\mathrm{C} 4-\mathrm{H} 4$ & 119.5 & $\mathrm{C} 5^{\prime}-\mathrm{C} 4^{\prime}-\mathrm{H} 4^{\prime}$ & 119.3 \\
\hline $\mathrm{C} 3-\mathrm{C} 4-\mathrm{H} 4$ & 119.5 & $\mathrm{C} 3^{\prime}-\mathrm{C} 4^{\prime}-\mathrm{H} 4^{\prime}$ & 119.3 \\
\hline $\mathrm{C} 4-\mathrm{C} 5-\mathrm{C} 6$ & $121.5(2)$ & $\mathrm{C} 4^{\prime}-\mathrm{C} 5^{\prime}-\mathrm{C} 6^{\prime}$ & $121.0(3)$ \\
\hline $\mathrm{C} 4-\mathrm{C} 5-\mathrm{H} 5$ & 119.2 & $\mathrm{C} 4^{\prime}-\mathrm{C} 5^{\prime}-\mathrm{H} 5^{\prime}$ & 119.5 \\
\hline $\mathrm{C} 6-\mathrm{C} 5-\mathrm{H} 5$ & 119.2 & $\mathrm{C} 6^{\prime}-\mathrm{C} 5^{\prime}-\mathrm{H} 5^{\prime}$ & 119.5 \\
\hline
\end{tabular}




\begin{tabular}{|c|c|c|c|}
\hline $\mathrm{C} 7-\mathrm{C} 6-\mathrm{C} 5$ & $117.5(2)$ & $\mathrm{C} 7^{\prime}-\mathrm{C} 6^{\prime}-\mathrm{C} 5^{\prime}$ & $117.8(3)$ \\
\hline $\mathrm{C} 7-\mathrm{C} 6-\mathrm{H} 6$ & 121.3 & $\mathrm{C} 7^{\prime}-\mathrm{C} 6^{\prime}-\mathrm{H} 6^{\prime}$ & 121.1 \\
\hline $\mathrm{C} 5-\mathrm{C} 6-\mathrm{H} 6$ & 121.3 & $\mathrm{C} 5^{\prime}-\mathrm{C} 6^{\prime}-\mathrm{H} 6^{\prime}$ & 121.1 \\
\hline $\mathrm{C} 6-\mathrm{C} 7-\mathrm{C} 2$ & $121.1(2)$ & $\mathrm{C} 6^{\prime}-\mathrm{C} 7^{\prime}-\mathrm{C} 2^{\prime}$ & $120.8(3)$ \\
\hline $\mathrm{C} 6-\mathrm{C} 7-\mathrm{C} 8$ & $129.4(2)$ & $\mathrm{C} 6^{\prime}-\mathrm{C} 7^{\prime}-\mathrm{C} 8^{\prime}$ & $129.4(2)$ \\
\hline $\mathrm{C} 2-\mathrm{C} 7-\mathrm{C} 8$ & $109.48(19)$ & $\mathrm{C} 2^{\prime}-\mathrm{C} 7^{\prime}-\mathrm{C} 8^{\prime}$ & $109.8(2)$ \\
\hline $\mathrm{O} 2-\mathrm{C} 8-\mathrm{N} 1$ & $112.16(17)$ & $\mathrm{O} 2^{\prime}-\mathrm{C} 8^{\prime}-\mathrm{N} 1^{\prime}$ & $112.0(2)$ \\
\hline $\mathrm{O} 2-\mathrm{C} 8-\mathrm{C} 7$ & $114.70(18)$ & $\mathrm{O} 2^{\prime}-\mathrm{C} 8^{\prime}-\mathrm{C} 7^{\prime}$ & $114.4(2)$ \\
\hline $\mathrm{N} 1-\mathrm{C} 8-\mathrm{C} 7$ & $101.68(17)$ & $\mathrm{N} 1^{\prime}-\mathrm{C} 8^{\prime}-\mathrm{C} 7^{\prime}$ & $101.48(19)$ \\
\hline $\mathrm{O} 2-\mathrm{C} 8-\mathrm{H} 8$ & 109.3 & $\mathrm{O} 2^{\prime}-\mathrm{C} 8^{\prime}-\mathrm{H} 8^{\prime}$ & 109.6 \\
\hline $\mathrm{N} 1-\mathrm{C} 8-\mathrm{H} 8$ & 109.3 & $\mathrm{~N} 1^{\prime}-\mathrm{C} 8^{\prime}-\mathrm{H} 8^{\prime}$ & 109.6 \\
\hline $\mathrm{C} 7-\mathrm{C} 8-\mathrm{H} 8$ & 109.3 & $\mathrm{C} 7^{\prime}-\mathrm{C} 8^{\prime}-\mathrm{H} 8^{\prime}$ & 109.6 \\
\hline $\mathrm{N} 1-\mathrm{C} 9-\mathrm{C} 10$ & $113.26(17)$ & $\mathrm{N} 1^{\prime}-\mathrm{C} 9^{\prime}-\mathrm{C} 10^{\prime}$ & $113.6(2)$ \\
\hline $\mathrm{N} 1-\mathrm{C} 9-\mathrm{H} 9 \mathrm{~A}$ & 108.9 & $\mathrm{~N} 1^{\prime}-\mathrm{C} 9^{\prime}-\mathrm{H} 9^{\prime} \mathrm{A}$ & 108.9 \\
\hline $\mathrm{C} 10-\mathrm{C} 9-\mathrm{H} 9 \mathrm{~A}$ & 108.9 & $\mathrm{C} 10^{\prime}-\mathrm{C} 9^{\prime}-\mathrm{H} 9^{\prime} \mathrm{A}$ & 108.9 \\
\hline N1-C9-H9B & 108.9 & $\mathrm{~N} 1^{\prime}-\mathrm{C} 9^{\prime}-\mathrm{H} 9^{\prime} \mathrm{B}$ & 108.9 \\
\hline $\mathrm{C} 10-\mathrm{C} 9-\mathrm{H} 9 \mathrm{~B}$ & 108.9 & $\mathrm{C} 10^{\prime}-\mathrm{C} 9^{\prime}-\mathrm{H} 9^{\prime} \mathrm{B}$ & 108.9 \\
\hline $\mathrm{H} 9 \mathrm{~A}-\mathrm{C} 9-\mathrm{H} 9 \mathrm{~B}$ & 107.7 & $\mathrm{H} 9^{\prime} \mathrm{A}-\mathrm{C} 9^{\prime}-\mathrm{H} 9^{\prime} \mathrm{B}$ & 107.7 \\
\hline $\mathrm{O} 3-\mathrm{C} 10-\mathrm{C} 9$ & $111.77(17)$ & $\mathrm{O} 3^{\prime}-\mathrm{C} 10^{\prime}-\mathrm{C} 9^{\prime}$ & $112.69(18)$ \\
\hline $\mathrm{O} 3-\mathrm{C} 10-\mathrm{C} 11$ & $101.29(16)$ & $\mathrm{O} 3^{\prime}-\mathrm{C} 10^{\prime}-\mathrm{C} 11^{\prime}$ & $100.79(17)$ \\
\hline $\mathrm{C} 9-\mathrm{C} 10-\mathrm{C} 11$ & $118.54(18)$ & $\mathrm{C} 9^{\prime}-\mathrm{C} 10^{\prime}-\mathrm{C} 11^{\prime}$ & $117.59(19)$ \\
\hline $\mathrm{O} 3-\mathrm{C} 10-\mathrm{C} 15$ & $100.25(15)$ & $\mathrm{O} 3{ }^{\prime}-\mathrm{C} 10^{\prime}-\mathrm{C} 15^{\prime}$ & $100.44(16)$ \\
\hline $\mathrm{C} 9-\mathrm{C} 10-\mathrm{C} 15$ & $115.28(18)$ & $\mathrm{C} 9^{\prime}-\mathrm{C} 10^{\prime}-\mathrm{C} 15^{\prime}$ & $114.75(19)$ \\
\hline $\mathrm{C} 11-\mathrm{C} 10-\mathrm{C} 15$ & $107.33(18)$ & $\mathrm{C} 11^{\prime}-\mathrm{C} 10^{\prime}-\mathrm{C} 15^{\prime}$ & $108.40(18)$ \\
\hline $\mathrm{C} 12-\mathrm{C} 11-\mathrm{C} 10$ & $106.5(2)$ & $\mathrm{C} 12^{\prime}-\mathrm{C} 11^{\prime}-\mathrm{C} 10^{\prime}$ & $106.32(19)$ \\
\hline $\mathrm{C} 12-\mathrm{C} 11-\mathrm{H} 11$ & 126.7 & $\mathrm{C} 12^{\prime}-\mathrm{C} 11^{\prime}-\mathrm{H} 11^{\prime}$ & 126.8 \\
\hline $\mathrm{C} 10-\mathrm{C} 11-\mathrm{H} 11$ & 126.7 & $\mathrm{C} 10^{\prime}-\mathrm{C} 11^{\prime}-\mathrm{H} 11^{\prime}$ & 126.8 \\
\hline $\mathrm{C} 11-\mathrm{C} 12-\mathrm{C} 13$ & $105.4(2)$ & $\mathrm{C} 11^{\prime}-\mathrm{C} 12^{\prime}-\mathrm{C} 13^{\prime}$ & $105.22(19)$ \\
\hline $\mathrm{C} 11-\mathrm{C} 12-\mathrm{H} 12$ & 127.3 & $\mathrm{C} 11^{\prime}-\mathrm{C} 12^{\prime}-\mathrm{H} 12^{\prime}$ & 127.4 \\
\hline $\mathrm{C} 13-\mathrm{C} 12-\mathrm{H} 12$ & 127.3 & $\mathrm{C} 13^{\prime}-\mathrm{C} 12^{\prime}-\mathrm{H} 12^{\prime}$ & 127.4 \\
\hline $\mathrm{O} 3-\mathrm{C} 13-\mathrm{C} 12$ & $102.11(16)$ & $\mathrm{O} 3^{\prime}-\mathrm{C} 13^{\prime}-\mathrm{C} 14^{\prime}$ & $113.05(18)$ \\
\hline $\mathrm{O} 3-\mathrm{C} 13-\mathrm{C} 14$ & $111.92(18)$ & $\mathrm{O} 3^{\prime}-\mathrm{C} 13^{\prime}-\mathrm{C} 12^{\prime}$ & $101.73(16)$ \\
\hline $\mathrm{C} 12-\mathrm{C} 13-\mathrm{C} 14$ & $125.30(19)$ & $\mathrm{C} 14^{\prime}-\mathrm{C} 13^{\prime}-\mathrm{C} 12^{\prime}$ & $124.03(19)$ \\
\hline $\mathrm{O} 3-\mathrm{C} 13-\mathrm{C} 16$ & $100.04(16)$ & $\mathrm{O} 3^{\prime}-\mathrm{C} 13^{\prime}-\mathrm{C} 16^{\prime}$ & $99.92(16)$ \\
\hline $\mathrm{C} 12-\mathrm{C} 13-\mathrm{C} 16$ & $107.30(18)$ & $\mathrm{C} 14^{\prime}-\mathrm{C} 13^{\prime}-\mathrm{C} 16^{\prime}$ & $106.76(17)$ \\
\hline $\mathrm{C} 14-\mathrm{C} 13-\mathrm{C} 16$ & $107.29(17)$ & $\mathrm{C} 12^{\prime}-\mathrm{C} 13^{\prime}-\mathrm{C} 16^{\prime}$ & $108.74(17)$ \\
\hline $\mathrm{N} 2-\mathrm{C} 14-\mathrm{C} 13$ & $103.49(17)$ & $\mathrm{N} 2^{\prime}-\mathrm{C} 14^{\prime}-\mathrm{C} 13^{\prime}$ & $103.46(17)$ \\
\hline $\mathrm{N} 2-\mathrm{C} 14-\mathrm{H} 14 \mathrm{~A}$ & 111.1 & $\mathrm{~N} 2^{\prime}-\mathrm{C} 14^{\prime}-\mathrm{H} 14 \mathrm{C}$ & 111.1 \\
\hline $\mathrm{C} 13-\mathrm{C} 14-\mathrm{H} 14 \mathrm{~A}$ & 111.1 & $\mathrm{C} 13^{\prime}-\mathrm{C} 14^{\prime}-\mathrm{H} 14 \mathrm{C}$ & 111.1 \\
\hline $\mathrm{N} 2-\mathrm{C} 14-\mathrm{H} 14 \mathrm{~B}$ & 111.1 & $\mathrm{~N} 2{ }^{\prime}-\mathrm{C} 14^{\prime}-\mathrm{H} 14 \mathrm{D}$ & 111.1 \\
\hline $\mathrm{C} 13-\mathrm{C} 14-\mathrm{H} 14 \mathrm{~B}$ & 111.1 & $\mathrm{C} 13^{\prime}-\mathrm{C} 14^{\prime}-\mathrm{H} 14 \mathrm{D}$ & 111.1 \\
\hline $\mathrm{H} 14 \mathrm{~A}-\mathrm{C} 14-\mathrm{H} 14 \mathrm{~B}$ & 109.0 & $\mathrm{H} 14 \mathrm{C}-\mathrm{C} 14^{\prime}-\mathrm{H} 14 \mathrm{D}$ & 109.0 \\
\hline $\mathrm{C} 16-\mathrm{C} 15-\mathrm{C} 10$ & $100.84(17)$ & $\mathrm{C} 16^{\prime}-\mathrm{C} 15^{\prime}-\mathrm{C} 10^{\prime}$ & $100.90(17)$ \\
\hline $\mathrm{C} 16-\mathrm{C} 15-\mathrm{H} 15 \mathrm{~A}$ & 111.6 & $\mathrm{C} 16^{\prime}-\mathrm{C} 15^{\prime}-\mathrm{H} 15 \mathrm{C}$ & 111.6 \\
\hline $\mathrm{C} 10-\mathrm{C} 15-\mathrm{H} 15 \mathrm{~A}$ & 111.6 & $\mathrm{C} 10^{\prime}-\mathrm{C} 15^{\prime}-\mathrm{H} 15 \mathrm{C}$ & 111.6 \\
\hline $\mathrm{C} 16-\mathrm{C} 15-\mathrm{H} 15 \mathrm{~B}$ & 111.6 & $\mathrm{C} 16^{\prime}-\mathrm{C} 15^{\prime}-\mathrm{H} 15 \mathrm{D}$ & 111.6 \\
\hline $\mathrm{C} 10-\mathrm{C} 15-\mathrm{H} 15 \mathrm{~B}$ & 111.6 & $\mathrm{C} 10^{\prime}-\mathrm{C} 15^{\prime}-\mathrm{H} 15 \mathrm{D}$ & 111.6 \\
\hline $\mathrm{H} 15 \mathrm{~A}-\mathrm{C} 15-\mathrm{H} 15 \mathrm{~B}$ & 109.4 & $\mathrm{H} 15 \mathrm{C}-\mathrm{C} 15^{\prime}-\mathrm{H} 15 \mathrm{D}$ & 109.4 \\
\hline
\end{tabular}




\begin{tabular}{|c|c|c|c|}
\hline $\mathrm{C} 17-\mathrm{C} 16-\mathrm{C} 15$ & $119.1(2)$ & $\mathrm{C} 17^{\prime}-\mathrm{C} 16^{\prime}-\mathrm{C} 15^{\prime}$ & $118.39(18)$ \\
\hline $\mathrm{C} 17-\mathrm{C} 16-\mathrm{C} 13$ & $101.38(17)$ & $\mathrm{C} 17^{\prime}-\mathrm{C} 16^{\prime}-\mathrm{C} 13^{\prime}$ & $101.21(16)$ \\
\hline $\mathrm{C} 15-\mathrm{C} 16-\mathrm{C} 13$ & $101.55(17)$ & $\mathrm{C} 15^{\prime}-\mathrm{C} 16^{\prime}-\mathrm{C} 13^{\prime}$ & $101.62(17)$ \\
\hline $\mathrm{C} 17-\mathrm{C} 16-\mathrm{H} 16$ & 111.2 & $\mathrm{C} 17^{\prime}-\mathrm{C} 16^{\prime}-\mathrm{H} 16^{\prime}$ & 111.5 \\
\hline $\mathrm{C} 15-\mathrm{C} 16-\mathrm{H} 16$ & 111.2 & $\mathrm{C} 15^{\prime}-\mathrm{C} 16^{\prime}-\mathrm{H} 16^{\prime}$ & 111.5 \\
\hline $\mathrm{C} 13-\mathrm{C} 16-\mathrm{H} 16$ & 111.2 & $\mathrm{C} 13^{\prime}-\mathrm{C} 16^{\prime}-\mathrm{H} 16^{\prime}$ & 111.5 \\
\hline $\mathrm{N} 2-\mathrm{C} 17-\mathrm{C} 16$ & $101.58(18)$ & $\mathrm{N} 2^{\prime}-\mathrm{C} 17^{\prime}-\mathrm{C} 16^{\prime}$ & $100.82(16)$ \\
\hline $\mathrm{N} 2-\mathrm{C} 17-\mathrm{H} 17 \mathrm{~A}$ & 111.5 & $\mathrm{~N} 2^{\prime}-\mathrm{C} 17^{\prime}-\mathrm{H} 17 \mathrm{C}$ & 111.6 \\
\hline $\mathrm{C} 16-\mathrm{C} 17-\mathrm{H} 17 \mathrm{~A}$ & 111.5 & $\mathrm{C} 16^{\prime}-\mathrm{C} 17^{\prime}-\mathrm{H} 17 \mathrm{C}$ & 111.6 \\
\hline $\mathrm{N} 2-\mathrm{C} 17-\mathrm{H} 17 \mathrm{~B}$ & 111.5 & $\mathrm{~N} 2^{\prime}-\mathrm{C} 17^{\prime}-\mathrm{H} 17 \mathrm{D}$ & 111.6 \\
\hline $\mathrm{C} 16-\mathrm{C} 17-\mathrm{H} 17 \mathrm{~B}$ & 111.5 & $\mathrm{C} 16^{\prime}-\mathrm{C} 17^{\prime}-\mathrm{H} 17 \mathrm{D}$ & 111.6 \\
\hline H17A-C17-H17B & 109.3 & $\mathrm{H} 17 \mathrm{C}-\mathrm{C} 17^{\prime}-\mathrm{H} 17 \mathrm{D}$ & 109.4 \\
\hline $\mathrm{C} 19-\mathrm{C} 18-\mathrm{C} 23$ & $120.8(2)$ & $\mathrm{C} 19^{\prime}-\mathrm{C} 18^{\prime}-\mathrm{C} 23^{\prime}$ & $120.5(2)$ \\
\hline $\mathrm{C} 19-\mathrm{C} 18-\mathrm{S} 1$ & $120.11(18)$ & $\mathrm{C} 19^{\prime}-\mathrm{C} 18^{\prime}-\mathrm{S} 1^{\prime}$ & $119.70(17)$ \\
\hline $\mathrm{C} 23-\mathrm{C} 18-\mathrm{S} 1$ & $119.01(17)$ & $\mathrm{C} 23^{\prime}-\mathrm{C} 18^{\prime}-\mathrm{S} 1^{\prime}$ & $119.76(17)$ \\
\hline $\mathrm{C} 20-\mathrm{C} 19-\mathrm{C} 18$ & $119.2(2)$ & $\mathrm{C} 20^{\prime}-\mathrm{C} 19^{\prime}-\mathrm{C} 18^{\prime}$ & $119.4(2)$ \\
\hline $\mathrm{C} 20-\mathrm{C} 19-\mathrm{H} 19$ & 120.4 & $\mathrm{C} 20^{\prime}-\mathrm{C} 19^{\prime}-\mathrm{H} 19^{\prime}$ & 120.3 \\
\hline $\mathrm{C} 18-\mathrm{C} 19-\mathrm{H} 19$ & 120.4 & $\mathrm{C} 18^{\prime}-\mathrm{C} 19^{\prime}-\mathrm{H} 19^{\prime}$ & 120.3 \\
\hline $\mathrm{C} 19-\mathrm{C} 20-\mathrm{C} 21$ & $121.0(2)$ & $\mathrm{C} 19^{\prime}-\mathrm{C} 20^{\prime}-\mathrm{C} 21^{\prime}$ & $120.9(2)$ \\
\hline $\mathrm{C} 19-\mathrm{C} 20-\mathrm{H} 20$ & 119.5 & $\mathrm{C} 19^{\prime}-\mathrm{C} 20^{\prime}-\mathrm{H} 20^{\prime}$ & 119.5 \\
\hline $\mathrm{C} 21-\mathrm{C} 20-\mathrm{H} 20$ & 119.5 & $\mathrm{C} 21^{\prime}-\mathrm{C} 20^{\prime}-\mathrm{H} 20^{\prime}$ & 119.5 \\
\hline $\mathrm{C} 22-\mathrm{C} 21-\mathrm{C} 20$ & $118.8(2)$ & $\mathrm{C} 22^{\prime}-\mathrm{C} 21^{\prime}-\mathrm{C} 20^{\prime}$ & $119.0(2)$ \\
\hline $\mathrm{C} 22-\mathrm{C} 21-\mathrm{C} 24$ & $119.4(2)$ & $\mathrm{C} 22^{\prime}-\mathrm{C} 21^{\prime}-\mathrm{C} 24^{\prime}$ & $120.3(2)$ \\
\hline $\mathrm{C} 20-\mathrm{C} 21-\mathrm{C} 24$ & $121.7(2)$ & $\mathrm{C} 20^{\prime}-\mathrm{C} 21^{\prime}-\mathrm{C} 24^{\prime}$ & $120.7(2)$ \\
\hline $\mathrm{C} 23-\mathrm{C} 22-\mathrm{C} 21$ & $121.2(2)$ & $\mathrm{C} 21^{\prime}-\mathrm{C} 22^{\prime}-\mathrm{C} 23^{\prime}$ & $120.9(2)$ \\
\hline $\mathrm{C} 23-\mathrm{C} 22-\mathrm{H} 22$ & 119.4 & $\mathrm{C} 21^{\prime}-\mathrm{C} 22^{\prime}-\mathrm{H} 22^{\prime}$ & 119.6 \\
\hline $\mathrm{C} 21-\mathrm{C} 22-\mathrm{H} 22$ & 119.4 & $\mathrm{C} 23^{\prime}-\mathrm{C} 22^{\prime}-\mathrm{H} 22^{\prime}$ & 119.6 \\
\hline $\mathrm{C} 22-\mathrm{C} 23-\mathrm{C} 18$ & $119.0(2)$ & $\mathrm{C} 22^{\prime}-\mathrm{C} 23^{\prime}-\mathrm{C} 18^{\prime}$ & $119.2(2)$ \\
\hline $\mathrm{C} 22-\mathrm{C} 23-\mathrm{H} 23$ & 120.5 & $\mathrm{C} 22^{\prime}-\mathrm{C} 23^{\prime}-\mathrm{H} 23^{\prime}$ & 120.4 \\
\hline $\mathrm{C} 18-\mathrm{C} 23-\mathrm{H} 23$ & 120.5 & $\mathrm{C} 18^{\prime}-\mathrm{C} 23^{\prime}-\mathrm{H} 23^{\prime}$ & 120.4 \\
\hline $\mathrm{C} 21-\mathrm{C} 24-\mathrm{H} 24 \mathrm{~A}$ & 109.5 & $\mathrm{C} 21^{\prime}-\mathrm{C} 24^{\prime}-\mathrm{H} 24 \mathrm{D}$ & 109.5 \\
\hline $\mathrm{C} 21-\mathrm{C} 24-\mathrm{H} 24 \mathrm{~B}$ & 109.5 & $\mathrm{C} 21^{\prime}-\mathrm{C} 24^{\prime}-\mathrm{H} 24 \mathrm{E}$ & 109.5 \\
\hline $\mathrm{H} 24 \mathrm{~A}-\mathrm{C} 24-\mathrm{H} 24 \mathrm{~B}$ & 109.5 & $\mathrm{H} 24 \mathrm{D}-\mathrm{C} 24^{\prime}-\mathrm{H} 24 \mathrm{E}$ & 109.5 \\
\hline $\mathrm{C} 21-\mathrm{C} 24-\mathrm{H} 24 \mathrm{C}$ & 109.5 & $\mathrm{C} 21^{\prime}-\mathrm{C} 24^{\prime}-\mathrm{H} 24 \mathrm{~F}$ & 109.5 \\
\hline $\mathrm{H} 24 \mathrm{~A}-\mathrm{C} 24-\mathrm{H} 24 \mathrm{C}$ & 109.5 & $\mathrm{H} 24 \mathrm{D}-\mathrm{C} 24^{\prime}-\mathrm{H} 24 \mathrm{~F}$ & 109.5 \\
\hline $\mathrm{H} 24 \mathrm{~B}-\mathrm{C} 24-\mathrm{H} 24 \mathrm{C}$ & 109.5 & $\mathrm{H} 24 \mathrm{E}-\mathrm{C} 24^{\prime}-\mathrm{H} 24 \mathrm{~F}$ & 109.5 \\
\hline
\end{tabular}

Hydrogen-bond geometry $\left(A,{ }^{\circ}\right)$

\begin{tabular}{|c|c|c|c|c|}
\hline$D-\mathrm{H} \cdots A$ & $D-\mathrm{H}$ & $\mathrm{H} \cdots A$ & $D \cdots A$ & $D-\mathrm{H} \cdots A$ \\
\hline $\mathrm{C} 8-\mathrm{H} 8 \cdots \mathrm{O} 3$ & 1.00 & 2.63 & $3.206(3)$ & 117 \\
\hline $\mathrm{C} 12-\mathrm{H} 12 \cdots \mathrm{O}^{\mathrm{i}}$ & 0.95 & 2.47 & $3.333(3)$ & 151 \\
\hline $\mathrm{C} 14-\mathrm{H} 14 A \cdots \mathrm{O} 5^{\prime \mathrm{ii}}$ & 0.99 & 2.62 & $3.506(3)$ & 149 \\
\hline $\mathrm{C} 23-\mathrm{H} 23 \cdots \mathrm{O} 5^{\prime \mathrm{ii}}$ & 0.95 & 2.41 & $3.195(3)$ & 139 \\
\hline $\mathrm{C} 14^{\prime}-\mathrm{H} 14 D^{\cdots} \mathrm{O} 4$ & 0.99 & 2.58 & $3.482(3)$ & 151 \\
\hline $\mathrm{C} 15^{\prime}-\mathrm{H} 15 C \cdots \mathrm{O} 2^{\mathrm{iii}}$ & 0.99 & 2.62 & $3.585(3)$ & 165 \\
\hline $\mathrm{C} 16^{\prime}-\mathrm{H} 16^{\prime} \cdots \mathrm{O} 4^{\prime \mathrm{iv}}$ & 1.00 & 2.53 & $3.422(3)$ & 149 \\
\hline $\mathrm{C} 19^{\prime}-\mathrm{H} 19^{\prime} \cdots \mathrm{O} 4$ & 0.95 & 2.34 & $3.078(3)$ & 134 \\
\hline
\end{tabular}


supporting information

\begin{tabular}{lllll}
$\mathrm{O} 2-\mathrm{H} 2 \cdots \mathrm{O}^{\mathrm{v}}$ & 0.92 & 1.85 & $2.756(2)$ & 172 \\
$\mathrm{O} 2{ }^{\prime}-\mathrm{H} 2^{\prime} \cdots \mathrm{O}^{\prime v \mathrm{vi}}$ & 0.90 & 1.95 & $2.840(3)$ & 171 \\
\hline
\end{tabular}

Symmetry codes: (i) $-x+1,-y+1,-z+1$; (ii) $x, y-1, z$; (iii) $x, y+1, z$; (iv) $-x+2,-y+2,-z+1$; (v) $-x+1,-y,-z+2$; (vi) $-x+2,-y+1,-z+2$. 